\title{
AFM Nanoindentation to Quantify the Mechanical Properties of Nano- and Micron-Sized Crystals of a Metal-Organic Framework Material
}

Zhixin Zeng and Jin-Chong Tan*

Multifunctional Materials \& Composites (MMC) Laboratory, Department of Engineering Science, University of Oxford, Parks Road, Oxford OX1 3PJ, United Kingdom

*jin-chong.tan@eng.ox.ac.uk

KEYWORDS: nanoindentation; atomic force microscopy (AFM); metal-organic framework (MOF); strain rate; interfacial sliding; fracture.

\begin{abstract}
The mechanical properties of individual nanocrystals and small micron-sized single crystals of metal-organic frameworks (MOFs), hitherto, cannot be measured directly by employing the conventional instrumented nanoindentation approach. Here we propose the application of atomic force microscopy (AFM)-based nanoindentation technique, equipped with a calibrated diamond cube-corner indenter tip to quantify the Young's modulus, hardness, adhesion energy, interfacial and fracture strengths of a zeolitic imidazolate framework (ZIF-8) porous material. We use ZIF-8 as a model MOF system to develop AFM nanoindentation leveraging the concept of
\end{abstract}


unloading strain rate, enabling us to critically assess the practicality and technical limitations of AFM to achieve quantitative measurements of fine-scale MOF crystals. We demonstrate the advantages of using a high unloading strain rate $\left(\dot{\varepsilon}>60 \mathrm{~s}^{-1}\right)$ to yield reliable force-displacement data in the few $\mu \mathrm{N}$ load range, corresponding to a shallow indentation depth of $\sim 10 \mathrm{~s} \mathrm{~nm}$. We found that the Young's moduli ( 3-4 GPa) determined by AFM nanoindentation of the nanocrystals $(<500 \mathrm{~nm})$ and micron-sized crystals $(\sim 2 \mu \mathrm{m})$ are in agreement with magnitudes derived previously from other techniques, namely instrumented nanoindentation and Brillouin spectroscopy (but these methods requiring large $100-\mu \mathrm{m}$ sized crystals), and also in line with density functional theory predictions of an idealized ZIF-8 crystal.

\section{Introduction}

Accurate characterization of the mechanical properties of emergent functional materials, such as metal-organic frameworks (MOFs), is central towards the engineering of practical applications. $^{1-3}$ MOFs are crystalline hybrid compounds, constructed from the self-assembly of metal clusters and organic linkers to yield porous framework structures exhibiting tunable physical and chemical properties. ${ }^{4-5}$ Several topical families of MOF materials are under intense study, encompassing zeolitic imidazolate frameworks (ZIFs) ${ }^{6}$ zirconium-based $\mathrm{UiOs}^{7}$ and MIL-140s, ${ }^{8}$ a vast variety of carboxylate-based MOFs (e.g. MILs, ${ }^{9}$ HKUST- $1,{ }^{10}$ and MOF-74s) ${ }^{11}$ and isoreticular frameworks (IRMOFs). ${ }^{12}$ There is considerable interest in the development of high-performance MOFs targeting gas separations and sequestration applications, ${ }^{13-14}$ with more recent emphasis shifting towards technological device applications in optoelectronics, low- $k$ dielectrics, chemical sensors and energy converters. ${ }^{15-17}$

Design, fabrication and deployment of the foregoing applications will depend on the availability of comprehensive mechanical properties information, plus a detailed understanding of 
MOF mechanics which remains lacking in the literature. ${ }^{1,18}$ There is, however, a growing body of work adopting theoretical methodologies, such as: density functional theory (DFT) to compute the full set of elastic constants of an ideal MOF crystal, ${ }^{19-22}$ and, by implementation of group theory ${ }^{23}$ to enable systematic studies of the structural flexibility of MOFs. Likewise, molecular dynamics $(\mathrm{MD})^{24-25}$ and $\mathrm{DFT}^{26}$ calculations have been used to interrogate the possible mechanisms that could be accommodating (irreversible) plastic deformation beyond the elastic regime. On the contrary, experimental methods for studying the mechanical properties of MOF crystals are far less established. To measure the Young's modulus (E) and hardness (H) properties, the MOF and crystal engineering community thus far has relied on the instrumented nanoindentation technique (for example, see refs.27-32), which requires access to a "large" single crystal of at least $\sim 100 \mu \mathrm{m}$ across. ${ }^{33}$ In practice, many of the interesting and important MOF materials can only be synthesized as small microcrystals or nanoparticles but often not as large single crystals,${ }^{34}$ such fine crystalline samples are not appropriate for mechanical characterization using the instrumented nanoindenter described above. For situations where only sub-micron crystals are achievable, nanoindentation experiments have been attempted on $\mu$ m-thick polycrystalline MOF films and coatings,${ }^{35-36}$ but these results are less quantitative because the indentation measurements are affected by the bulk substrate underneath (causing $\mathrm{E}$ and $\mathrm{H}$ values to artificially rise with indentation depth, see for example ref.37). Nanoscratch experiments have also been demonstrated for the characterization of the adhesion behavior of polycrystalline MOF films, again the data obtained so far are semiquantitative..$^{38-39}$

While atomic force microscopy (AFM)-based nanoindentation method is frequently used to study the mechanical behavior of soft solids, e.g. polymers, hydrogels and biological samples, ${ }^{40-}$ ${ }^{41}$ the applicability of AFM nanoindentation to the field of MOF-type materials has not yet been 
demonstrated. Given the capability of the AFM instrument to control a significantly smaller indentation load (force sensitivity $<0.05 \mathrm{nN}$ ), shallower indentation depth (displacement sensitivity $\lesssim 0.05 \mathrm{~nm})^{42}$ and effective contact volume, this combination will offer us the unique opportunity to probe local mechanical properties of small crystals and thin-film samples previously inaccessible via the "standard" instrumented nanoindentation approach. Although some of the "standard" approaches have nanoscale sensitivity, in practice it is not feasible to perform nanoindentation as subtle as AFM, whose load sensitivity is on the picoNewton $(\mathrm{pN})$ level. AFM nanoindentation also enables high-resolution 3D imaging to map the surface height topography and provides in situ quantification of the shape of the residual indents. However, quantitative interpretation of the AFM nanoindentation data is not straightforward, because the instrumentaland sample-related conditions could impact the precision of the force-displacement measurements to different extents. Amongst the foreseeable challenges are: (i) Distorted force-displacement curve and erroneous contact area might be the result of a blunt/damaged tip combined with the intrinsic compliance of the AFM cantilever probe; (ii) A smooth and flat sample surface is important for accurate contact area determination (the same condition applies to instrumented nanoindentation but at a different length scale); (iii) An AFM probe with a larger tip angle (e.g. a cube-corner) is generally used for indentation but at the expense of reduced resolution in 3D imaging; (iv) Indentation-triggered effects, such as "pile-up" and "sink-in" at the periphery of the indenter (also encountered in standard nanoindentation), ${ }^{33}$ creep/viscoelasticity and adhesion interactions during indenter unloading ${ }^{43}$ could affect the quality and reproducibility of the data.

In this paper, we present the first use of AFM nanoindentation to accomplish quantitative characterization of the mechanical properties of ZIF-8 $[\mathrm{Zn}(\mathrm{mIM}) 2, \mathrm{mIM}=2$-methylimidazolate $]{ }^{6}$ which features a porous sodalite framework architecture (Figure 1). We have performed accurate 
measurements on the sub-micron $(<500 \mathrm{~nm})$ and isolated micron-sized $(\sim 1-2 \mu \mathrm{m})$ crystals of ZIF-8, whose averaged size is about one thousand times smaller ${ }^{28,44}$ than those quantifiable utilizing the established instrumented nanoindentation approach. By monitoring the strain rates of the AFM indenter tip during the indentation process, we demonstrate the strategy to determine reliable Young's modulus and hardness values using very small crystal samples. Furthermore, we elucidated the effects of adhesion forces developing at the tip-to-sample interface, and explained the failure modes and estimated fracture strengths underpinning the ZIF-8 crystals.

\section{Experimental Section}

\subsection{Synthesis of nanocrystals of ZIF-8}

The sub-micron sized crystals of ZIF-8 used in this study were typically of 300 to $500 \mathrm{~nm}$ in diameter, as characterized in Figure 1 and in Supporting Information (Figure S1). Such ZIF-8 nanocrystals were synthesized by dissolving $4.5 \mathrm{mmol}$ of zinc nitrate hexahydrate $\left(\mathrm{Zn}\left(\mathrm{NO}_{3}\right)_{2} \cdot 6 \mathrm{H}_{2} \mathrm{O}, 98 \%\right.$, Sigma-Aldrich), and $13.5 \mathrm{mmol}$ 2-methylimidazole (98\%, Sigma-Aldrich) in $60 \mathrm{~mL}$ methanol, respectively. The precursors were sonicated until the solutes were completely dissolved, then combined and heated in an oven at $40^{\circ} \mathrm{C}$ for $1 \mathrm{hr}$, resulting in the formation of a white colloidal suspension comprising ZIF-8 nanocrystals. Sonication of the mixture solution was avoided to prevent growth of ZIF-8 clusters; likewise, increasing the reaction time might lead to the formation of unwanted polycrystalline aggregates. Nanocrystals were isolated by centrifugation at $8000 \mathrm{rpm}$ for $10 \mathrm{mins}$, then the clear solution was removed before re-dispersing the extracted ZIF-8 in fresh methanol. The washing step was repeated 3 times to ensure the complete removal of excess reactants.

ZIF-8 nanocrystals were used to prepare a concentrated suspension in methanol $(\sim 5 \mathrm{~mL})$, which was then drop casted onto a dust-free glass substrate held inclined at $\sim 10^{\circ}$ to $20^{\circ}$ to the 
vertical direction. Using this approach, we achieved a polycrystalline thin film of ZIF-8 (Figure 1a) with a nominal thickness of $\sim 2 \mu \mathrm{m}$ and a root-mean-square (rms) roughness of $\sim 30 \mathrm{~nm}$ (measured by a non-contact optical profilometer, Alicona InfiniteFocus). Figure $1 \mathrm{~b}$ shows the X-ray diffraction pattern obtained from the drop-cast thin film, confirming the high crystallinity and phase purity of the ZIF-8 nanocrystals.

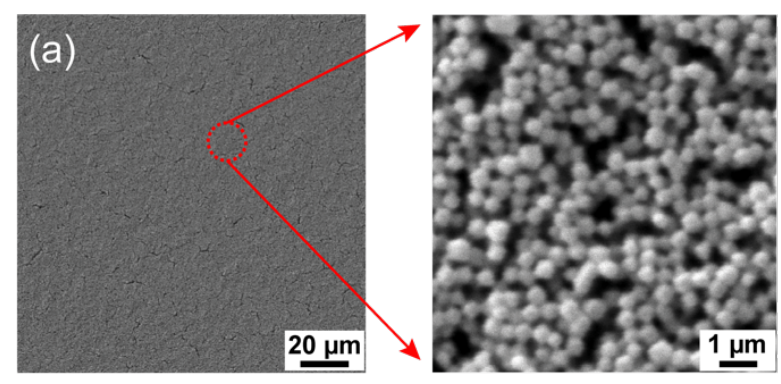

(c)
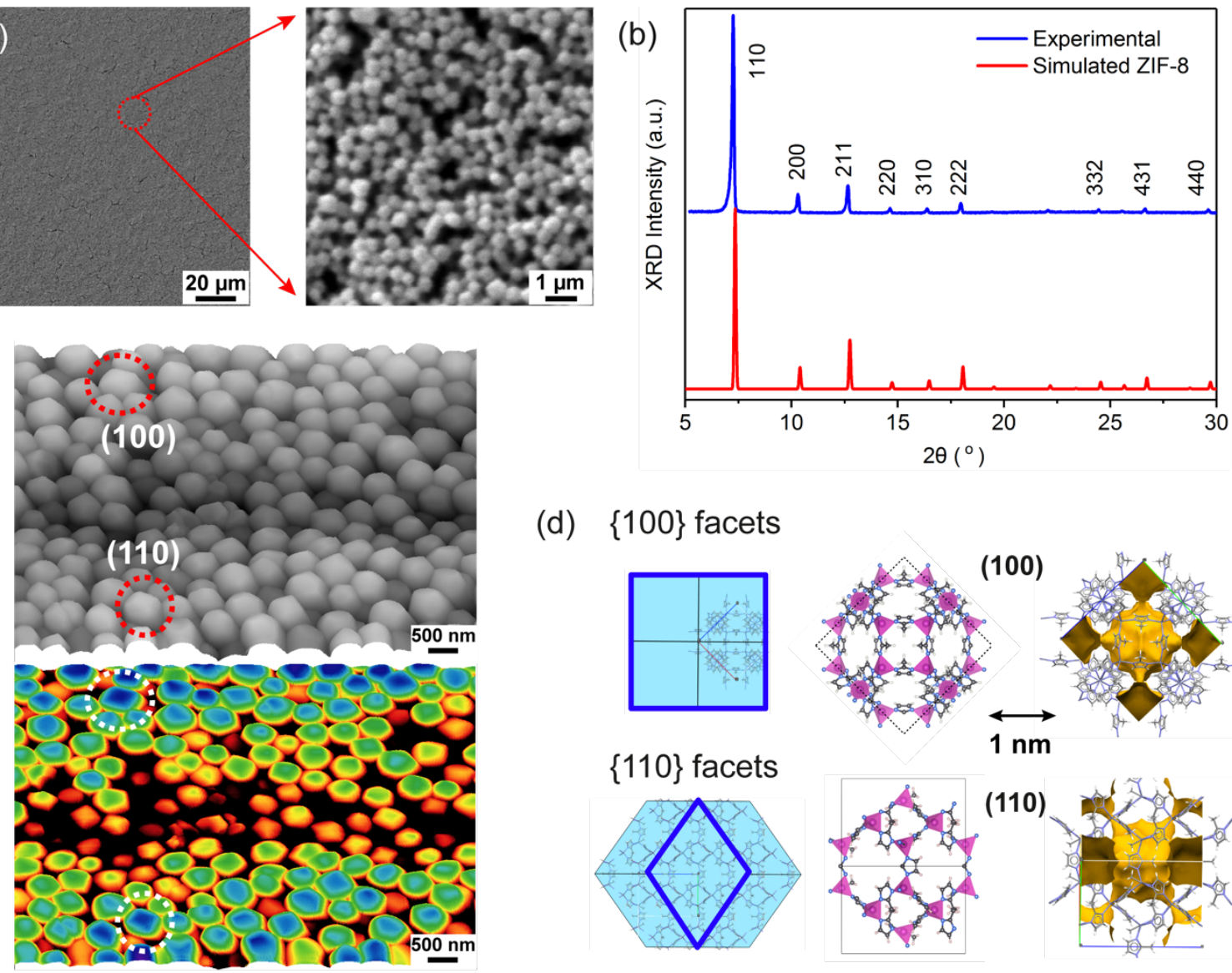

(d) $\{100\}$ facets

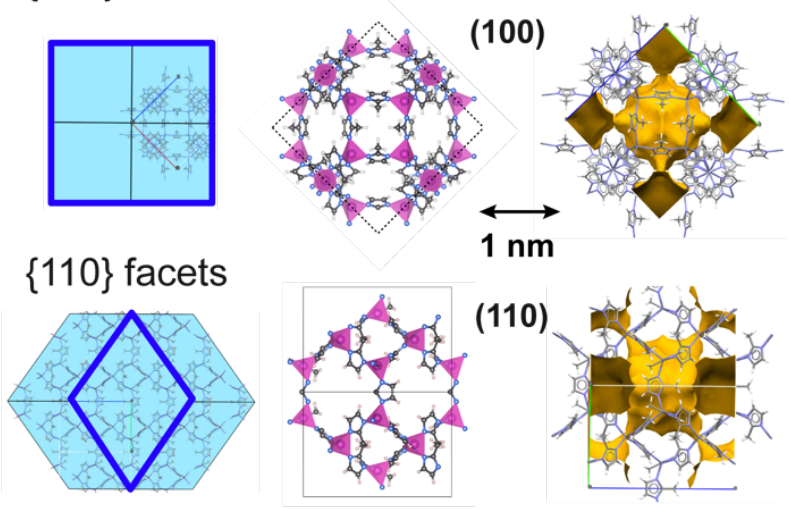

Figure 1. (a) Scanning electron microscope (SEM) images of a polycrystalline thin-film coating prepared by drop casting of uniform ZIF-8 nanocrystals. (b) X-ray diffraction pattern of the drop-cast ZIF-8 film, matching all the simulated $(h k l)$ Bragg peaks of an ideal ZIF-8 structure. Intense diffraction attributed to the $\{110\}$ crystallographic planes. (c) AFM height topography acquired using an imaging silicon tip (Tap300Al-G). Contour maps revealing the crystallographic 
orientation of the nanosized crystals, where the $\{110\}$-oriented rhombic facets are prevalent and with the occasional detection of the $\{100\}$ square facets. (d) Crystal habits of ZIF-8 crystal, where in this study the majority consisted of rhombic dodecahedron with exposed $\{110\}$-oriented facets, indexed on the basis of the crystal facets measured via AFM imaging technique (depicted in (c)). Porous crystalline framework architecture of ZIF-8, where the purple tetrahedron represents the $\mathrm{ZnN}_{4}$ coordination environment bridged by the 2-methylimidazolate (mIM) organic linkages; (far right) the yellow surfaces designate the solvent accessible volume (porosity) in a cubic unit cell of ZIF-8 viewed down the [100]- and [110]-axes, respectively.

\subsection{Synthesis of micron crystals of ZIF-8}

The micron-sized crystals of ZIF-8 employed in this study had a mean diameter of 1-2 $\mu \mathrm{m}$, synthesized using a solvothermal method. Two precursor solutions were prepared by dissolving $1.34 \mathrm{~g}$ (4 mmol) of zinc nitrate hexahydrate, and $0.334 \mathrm{~g}(4 \mathrm{mmol})$ of 2 -methylimidazole in $40 \mathrm{~mL}$ of methanol, respectively. A mixture of $80 \mathrm{~mL}$ was obtained by combining the two precursor solutions, which was then left (without stirring) for $24 \mathrm{hr}$ at room temperature. The product comprising micron-sized ZIF-8 crystals (see Figure 7a-c) was washed several times with methanol, centrifuged, and then dried overnight in a vacuum oven at $90^{\circ} \mathrm{C}$.

\subsection{AFM nanoindentation using a diamond-tipped cantilever probe}

Atomic force microscopy (AFM) nanoindentation experiments were performed using the Veeco Dimension 3100 instrument operating under the indentation mode, equipped with a Bruker PDNISP probe. The AFM probe consists of a $350 \mu \mathrm{m}$ long stainless steel cantilever, at the end of 
which mounted a cube-corner diamond indenter tip. Its spring constant and contact sensitivity have been calibrated by the manufacturer, and given as $152.285 \mathrm{~N} / \mathrm{m}$ and $256.6 \mathrm{~nm} / \mathrm{volt}$, respectively.

Establishing the accurate geometry of the indenter tip is extremely important for enabling quantitative measurements of the fine-scale mechanical properties. Herein to ascertain the actual geometry of the diamond tip, we have adopted the blind tip estimation algorithm developed by Villarrubia ${ }^{45}$ which has been validated by experiments. ${ }^{46} \mathrm{We}$ applied this algorithm as implemented in the Gwyddion software ${ }^{47}$ see further details described in Supporting Information (Figure S2-S3). Figure 2 shows the geometry estimated for the cube-corner indenter used in this study: front angle $\angle \mathrm{ADB}=58.2422^{\circ}$; back angle $\angle \mathrm{CDB}=35.1542^{\circ}$; side angle $\angle \mathrm{CEF}=49.1985^{\circ}$. Based on the indenter tip geometry established above, the equivalent conical angle $2 \theta$ was found to be $91.4667^{\circ}$, with which the nominal contact area as a function of the indentation depth, $A(h)$, can be determined. 


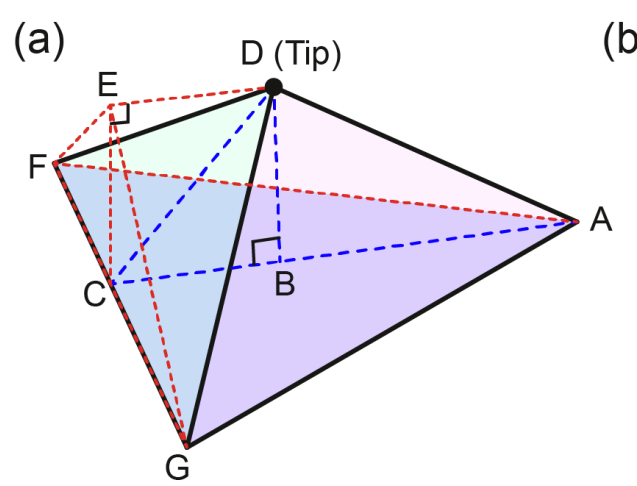

(b)

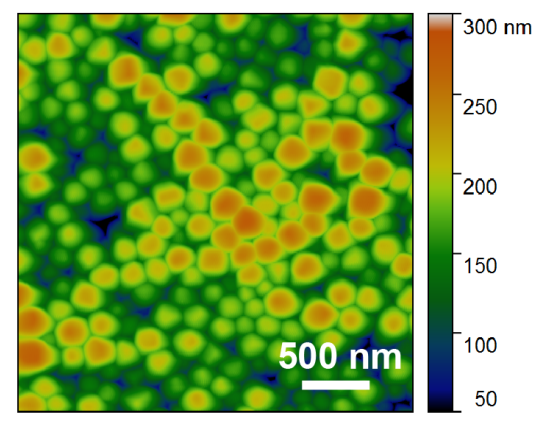

(c)

(d)
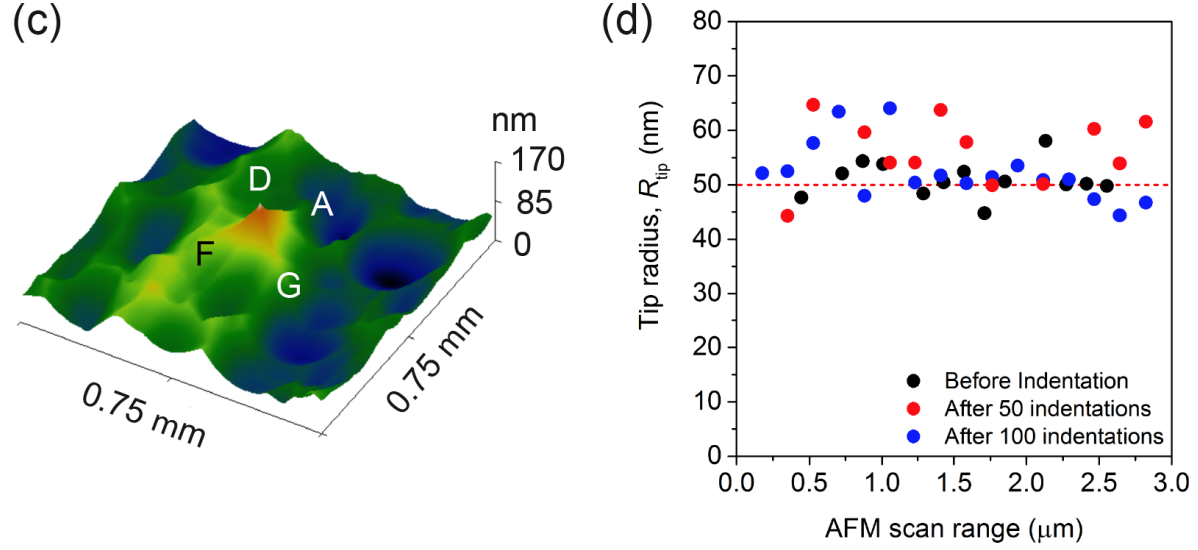

Figure 2. (a) Pyramidal geometry of the cube-corner indenter tip. (b) AFM height topography of a sample of ZIF-8 thin film imaged by the Bruker PDNISP indenter probe, which later served as the input data for the blind tip estimation algorithm. (c) Height topography image of the indenter tip geometry, showing an example reconstruction obtained using the Villarrubia algorithm. (d) The radius of the diamond indenter apex was monitored at different stages of the indentation study, where it was determined that $R_{\text {tip }}$ is $\sim 50 \pm 10 \mathrm{~nm}$.

\subsection{AFM nanoindentation methodology and $\boldsymbol{P}$ - $\boldsymbol{h}$ data analysis}

The AFM nanoindentation measurements yield a set of indenter load-vs-displacement $(P-h)$ curves, from which the mechanical properties particularly the Young's modulus $(E)$ and hardness $(H)$ can be quantified. In this study, we adopted the Oliver and Pharr (O\&P) method ${ }^{48}$ (see Supporting Information, §9), which is applicable to the cube-corner indenter geometry. Our approach, therefore, is different from the common methods typically implemented for the analysis 
of AFM nanoindentation data, namely the Hertz, JKR (Johnson-Kendall-Roberts) and DMT (Derjaguin-Muller-Toporov) contact models, ${ }^{49}$ which simplified the analysis assuming a spherical-tip indenter geometry. The aforementioned methods (O\&P included) share certain limitations: firstly, they assume an isotropic material response ${ }^{50}$ and disregard the effects of pile-up and/or sink-in; ${ }^{33}$ secondly, they disregard any time-dependent deformation mechanisms, such as creep or viscoelastic effects.

Importantly, herein we demonstrate that unloading strain rate of the indenter tip, $\dot{\varepsilon}$, is a leading parameter to control during AFM nanoindentation of compliant materials, to allow reliable measurements of the Young's modulus of nano- and micron-sized ZIF-8 crystals. Moreover, we show that the loading and unloading strain rates can be tuned by varying the AFM scan rate $(V)$ and the cantilever probe deflection $(\delta)$ in the Veeco Dimension 3100 AFM we used for this study (since this is a basic AFM setup, our proposed approach will be easily adapted to other more sophisticated systems). The strain rate at the maximum indentation depth can be determined from $\dot{\varepsilon}=\dot{P} / P_{\max }$ (see detailed explanations in Supporting Information $\S 3$ ), ${ }^{51}$ coinciding with the initial point when the indenter starts to unload. By applying this AFM nanoindentation methodology, we have measured the local mechanical properties of MOF crystals over a wide range of unloading strain rates. In the meantime, in order to address the influence of adhesion forces on mechanical properties measurement, we also develop an improved method that successfully reduces the deviations of the Young's moduli of micron-sized ZIF-8. First attempts in the fine-scale characterization of fracture mechanism as well as interfacial strength between ZIF-8 polycrystals has been undertaken, by virtue of the superior sensitivity conferred by the AFM-based nanoindentation approach. 


\section{Nanoscale mechanical properties of ZIF-8}

\subsection{AFM nanoindentation of ZIF-8 nanocrystals}

We performed AFM nanoindentation experiments in accordance with the strain-rate controlled approach outlined, to study thin film samples (Figure 1) prepared via drop casting of (sub-micron sized) ZIF-8 nanocrystals. We have accomplished indentation measurements with a surface penetration depth $(h)$ ranging from $10 \mathrm{~nm}$ to $80 \mathrm{~nm}$, corresponding to an applied load $(P)$ of between $0.3 \mu \mathrm{N}$ and $15 \mu \mathrm{N}$. Figure 3 a shows a set of representative $P$ - $h$ curves for indentation depths of just $\sim 10 \mathrm{~nm}$ to $30 \mathrm{~nm}$ onto the top facets of the individual nanocrystals, whose size is $c a$. 300 to $500 \mathrm{~nm}$. These shallow indentations lie within the recommended $10 \%$ rule of the sample thickness ${ }^{52}$ vis-á-vis the size of the rhombic dodecahedron nanocrystals of ZIF-8, so as to avoid any substrate effects. Figure $3 \mathrm{~b}$ shows the relatively deeper $P-h$ indentation curves, for a surface penetration depth of up to $h_{\max } \sim 60 \mathrm{~nm}$. We found that direct 3-D mapping of the residual indents using AFM topographic imaging was not attainable for the shallow indentation measurements described above, because of two factors: (i) the large elastic recovery upon tip unloading is clearly

evident in Figure $3 \mathrm{a}$ where $h_{\text {final }} \lesssim 10 \mathrm{~nm}$, and (ii) the 50 -nm tip radius $\left(R_{\text {tip }}\right)$ of the diamond cube-corner probe (Figure 2d) is not sufficiently sharp for imaging the very shallow residual indents ( few nm) after elastic recovery, because $h_{\text {final }} \leqslant R_{\text {tip }}$. 

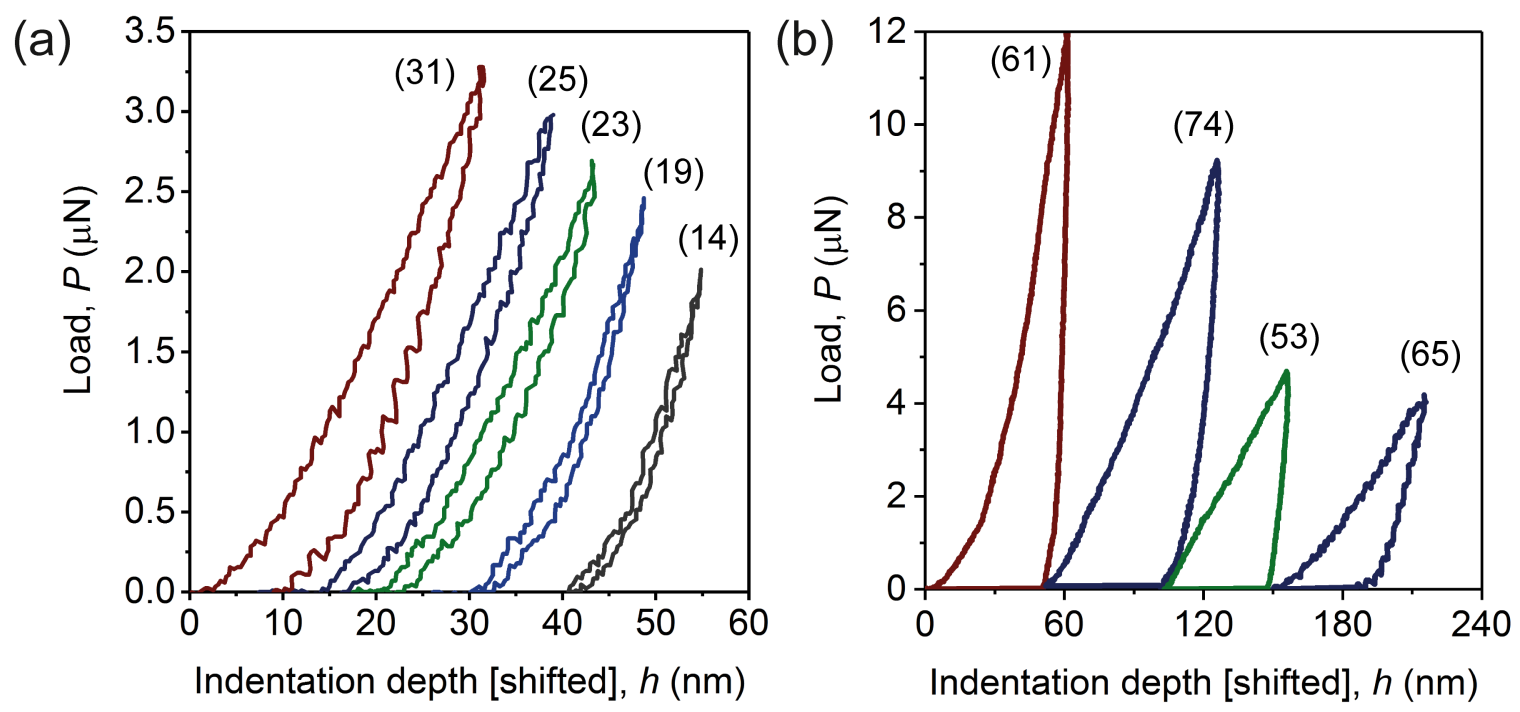

Figure 3. AFM nanoindentation force-displacement $(P-h)$ curves of individual nanocrystals of ZIF-8 showing (a) the shallow indents with $h_{\max } \lesssim 30 \mathrm{~nm}$, and (b) the relatively deeper indents with $h_{\max } \sim 60 \mathrm{~nm}$. Note that the displacement axis on the abscissa has been shifted for clarity. Note that the numbers in brackets represent the maximum indentation depths (in $\mathrm{nm}$ ).

We established that, as shown in Figure 4, there are two primary AFM instrument parameters controlling the indentation strain rate $(\dot{\varepsilon})$, specifically: the scan rate of the cantilever probe ( $V$ is speed of indenter spanning the load-unload motion of the piezo-electronics), and its pre-set vertical deflection $(\delta)$ of the cantilever tip. In this work, we could control the load-unload strain rates $(\dot{\varepsilon}=\dot{P} / P)$ of the probe for each individual indentation test (Figure S4 in Supporting Information). It can be seen in Figure 4a-b that a high unloading strain rate approaching $140 \mathrm{~s}^{-1}$ could be achieved by raising the scan rate $V$ to $\sim 1 \mathrm{~Hz}$, while reducing the cantilever deflection $\delta$ to under $10 \mathrm{~s} \mathrm{~nm}$. There is a reasonably good control over the unloading strain rates of the AFM indenter; for example, Figure 4c shows the upward trend of $\dot{\varepsilon}$ with different pre-set scan rates, whereas an inversed scaling relation can be observed in Figure $4 \mathrm{~d}$ with respect to the cantilever deflection of varying scan rates. Together, our results reveal that optimal combinations of $V$ and $\delta$ 
parameters do exist, as presented in the contour plot of Figure 4e. In essence when indenting soft materials and/or compliant interfaces, we should maximize the unloading strain rates to yield a $\dot{\varepsilon}$ high enough to surpass any unwanted creep effects (to be elucidated below), as well as to prevent extensive fracture of porous framework that will result in an erroneous contact area attributed to distorted $P$ - $h$ curves (see $\$ 5$ in Supporting Information). In this study, we demonstrate that by maintaining a high unloading strain rate (Figure 4), it is possible to alleviate the negative-gradient test segment of unloading curves when measuring small-sized ZIF-8 crystals. 

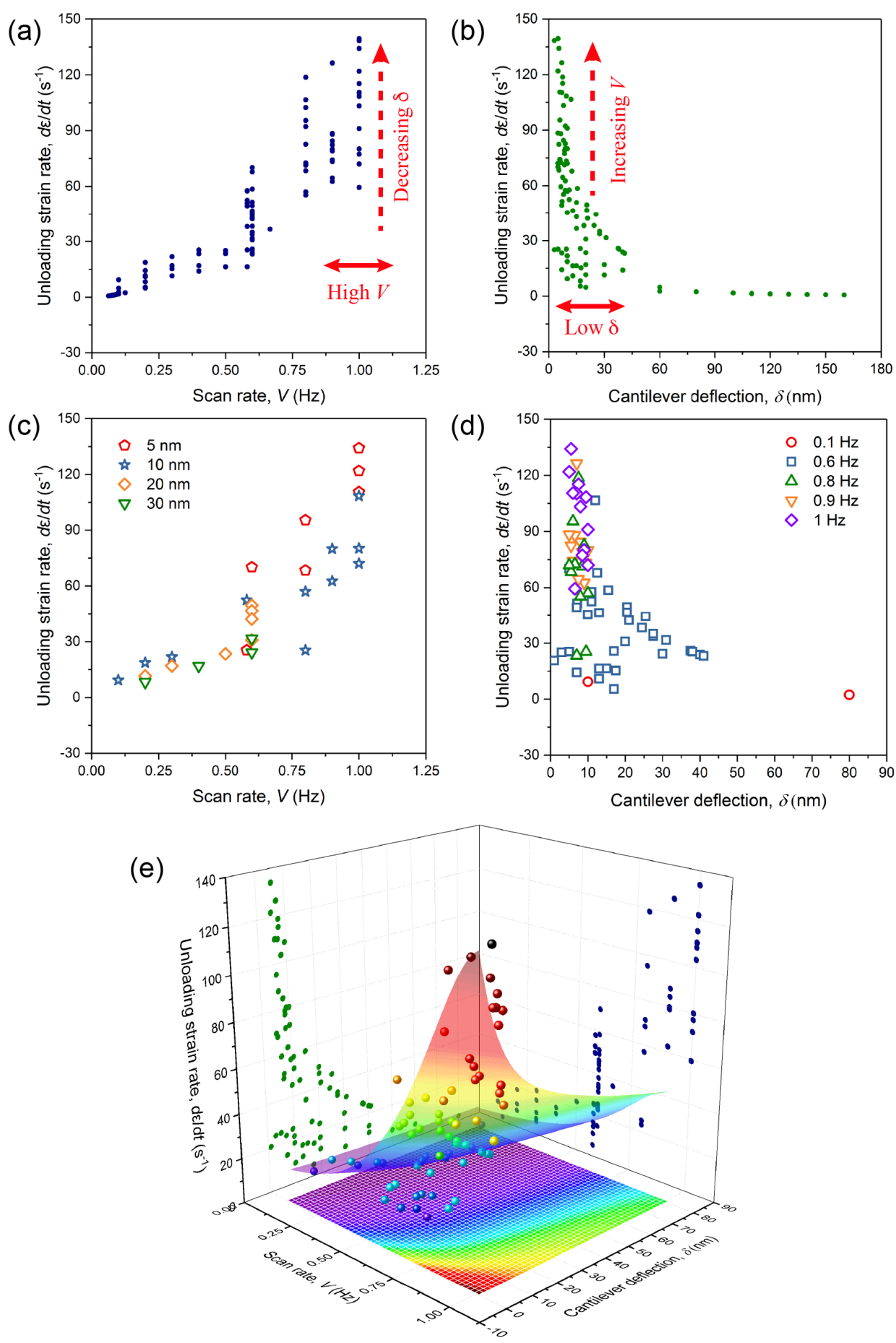

Figure 4. (a) Unloading strain rate $(\dot{\varepsilon})$ of the AFM indenter probe as a function of the cantilever scan rate $V$. (b) $\dot{\varepsilon}$ as a function of the cantilever deflection $\delta$. Data plotted in (a) and (b) were obtained from a total of 72 measurements conducted on individual ZIF-8 nanocrystals like those 
depicted in Figure 1. Selected data highlighting the effects of increasing (c) the cantilever deflection from 5-30 nm, and (d) the scan rate between 0.1-1 Hz. (e) Contour plot of all 72 indention experiments, showing the combined effects of different scan rate and cantilever deflection, in which the red region marks the desired strain rates for overcoming the creep effects upon unloading of the indenter tip.

We have determined the Young's modulus $(E)$ of the ZIF-8 nanocrystals by analyzing the individual $P$ - $h$ curves, using the equations set forth in Supporting Information $§ 9$. The results we obtained from a total of 72 indentation experiments are plotted in Figure 5, allowing us to understand the effects that the unloading strain rate $(\dot{\varepsilon})$ and/or the maximum indentation depth $\left(h_{\max }\right)$ might have on the quantified values of $E$. In fact, the Young's modulus of ZIF-8 is well-established in the literature, both using instrumented nanoindentation method of a significantly larger $\sim 150 \mu \mathrm{m}$ crystal $\left(E_{\{110\}}=3.2 \mathrm{GPa}\right)^{28}$ and by Brillouin spectroscopy technique validated by density functional theory $\left(E_{\{110\}} \sim 3 \mathrm{GPa}\right.$ and $E_{V R H}=3.15 \mathrm{GPa}$, the latter is the Voigt-Reuss-Hill averaged value for a polycrystalline ZIF-8 sample). The cubic symmetry of ZIF-8 also exhibits elastic anisotropy, where $E_{\max } \sim 3.8 \mathrm{GPa}$ and $E_{\min } \sim 2.8 \mathrm{GPa}$ corresponding to the Young's moduli of the $\{100\}$ - and $\{111\}$-oriented crystal facets, respectively (NB. readers should consult ref. ${ }^{44}$ about the source of elastic anisotropy and associated mechanisms).

In the light of this, we have chosen ZIF-8 as a "model system" to develop the AFM nanoindentation technique based on the concept of unloading strain rate, in order to critically assess the feasibility and technical limits of this AFM approach, and, to understand its applicability to probe fine-scale MOF crystals. Notably it can be seen in Figure 5a that, when the applied $\dot{\varepsilon}$ is exceeding $\sim 60 \mathrm{~s}^{-1}$ the measured $E$ values start to converge towards the known literature value of 
$\sim 3 \mathrm{GPa}$. In contrast, it is evident that there are major overestimations (up to 900\%) of the elastic moduli when the $\dot{\varepsilon}$ is slower than $25 \mathrm{~s}^{-1}$. Particularly we found that when $\dot{\varepsilon}<3 \mathrm{~s}^{-1}$, the incipient unloading curves began to develop a negative-gradient segment indicating that the creep rate is becoming significantly higher than the indenter unloading rate (Figure S6 in Supporting Information); notably when creep dominates the initial unloading stage this will result in a spuriously high Young's modulus value. The adverse effects of creep deformation to AFM nanoindentation measurements are well recognized in viscoelastic solids and polymers. ${ }^{53-54}$
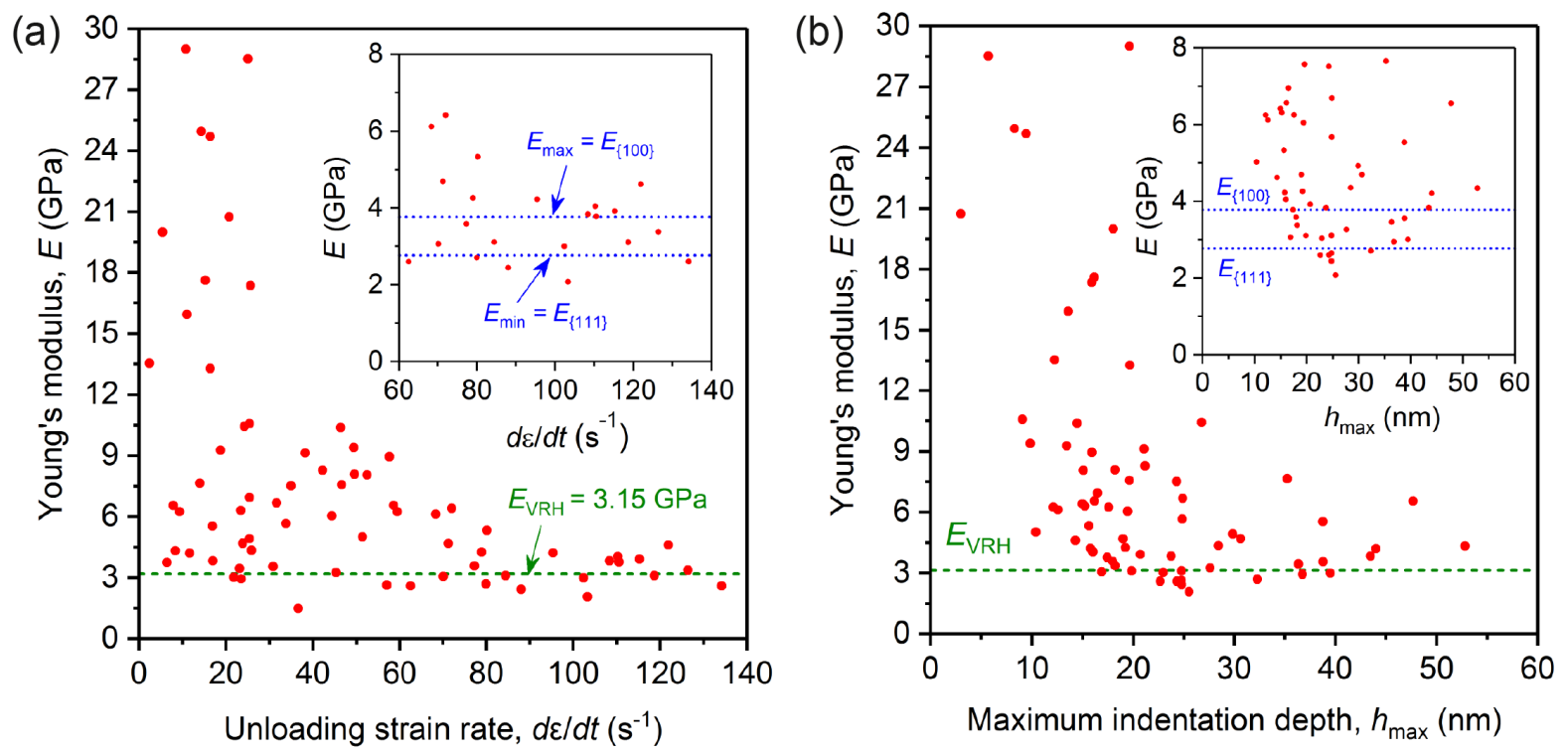

Figure 5. (a) Young's modulus, $E$, plotted as a function of the (initial) unloading strain rate of the indenter tip, where a higher unloading strain rate stabilizes the magnitude of the measured moduli coinciding with the literature value for ZIF-8 (the green line is the literature value ${ }^{44}$ assuming an isotropic polycrystalline ZIF-8 sample). The inset compares the AFM results against the reported upper bound $E_{\{100\}}$ and the lower bound $E_{\{111\}}$ of a ZIF-8 single crystal, arising from the effects of elastic anisotropy. (b) The variation of E plotted as a function of the maximum indentation depth. 
As shown in Figure $5 \mathrm{~b}$ akin to instrumented nanoindentation data, the results of Young's modulus acquired from the AFM nanoindentation method can also be presented as a function of the maximum indentation depth, i.e. $h_{\max }$, determined at the point of indenter unloading. Although a reasonable agreement to the literature value $(E \sim 3 \mathrm{GPa})$ can be attained when $h_{\max }$ is much deeper than $\sim 30 \mathrm{~nm}$, we observed that the correlation to the unloading strain rate is more superior (Figure 5a). This is because the unloading strain rate is a more versatile parameter to be tuned when indenting compliant materials susceptible to creep deformation (such as an aggregate of MOF nanocrystals), where $\dot{\varepsilon}$ can be varied to obtain a consistently high value ( $\left.>60 \mathrm{~s}^{-1}\right)$ across a range of indentation depths.

The hardness $(H)$ property of the ZIF-8 nanocrystals can be obtained by definition: $H=P_{\text {max }} / A_{\text {contact }}$. Because the contact area established under the maximum load is determined only by the maximum indentation depth $\left(h_{\max }\right)$ of the loading test segment, hardness derived from AFM nanoindentation is thereby independent of the unloading strain rate. The hardness results are given in Figure 6, showing a convergence towards the literature value for indentation depths of much greater than $\sim 30 \mathrm{~nm}$. This is a remarkable improvement given that the reported hardness ( $H \sim 500 \mathrm{MPa})$ required the use of a considerably larger single crystal $(150 \mu \mathrm{m})$ of ZIF-8, whose results were averaged over a depth of 100-1000 nm. ${ }^{28}$ Significantly, in this work we have indented nanocrystals (300-500 nm) which are about 1000× smaller and yet we have established comparable $H$ values for an indentation depth of between $\sim 30-80 \mathrm{~nm}$. The rapid rise in hardness values for depths below $\sim 30 \mathrm{~nm}$ might be linked to the indentation size effects, ${ }^{55-56}$ evidenced also in instrumented indentation studies ${ }^{57}$ (but typically detected at $<200 \mathrm{~nm}$, see Figure 6 inset). 
For the first time, the AFM approach has offered us the unique opportunity to selectively indent individual sub-micron sized MOF crystals, hitherto, not possible utilizing an instrumented (standard) nanoindenter setup that will require the preparation of a considerably larger crystal size, of at least $\sim 100 \mu \mathrm{m} .{ }^{29,58}$ We show that direct quantification of the AFM nanoindentation data is possible to achieve reliable measurements of $E$ and $H$ values, consistent with instrumented nanoindentation tests but requiring only crystals that are about 1000x smaller. There are other advantages for using AFM-based nanoindentation to study MOF mechanics: the load sensitivity of the AFM is about three orders of magnitude better than that of an instrumented nanoindenter, ${ }^{42}$ hence enabling precise deformation of pliant porous structures such as MOFs. Moreover, because the translation stages of instrumented indenters are screw-driven, their spatial positioning accuracy is limited to $\sim 1 \mu \mathrm{m}$, thereby not suitable to accurately locate and probe standalone nanoparticles and sub-micron scale crystals.

It is worth emphasizing that successful AFM nanoindentation of sub-micron crystals (nanocrystals) will depend on several factors: (a) High surface roughness of polycrystalline films and coatings could affect the stability of the indentation measurement, therefore minimum sample roughness is desirable to avoid any error in contact area determination; (b) Accurate calibration of the indenter geometry and tip radius is crucially important to prevent erroneous results when analyzing the Young's modulus and hardness based on the $P$ - $h$ curves. (c) High unloading strain rates are necessary to overcome time-dependent deformation, such as creep effects that may lead to spurious stiffness values. 


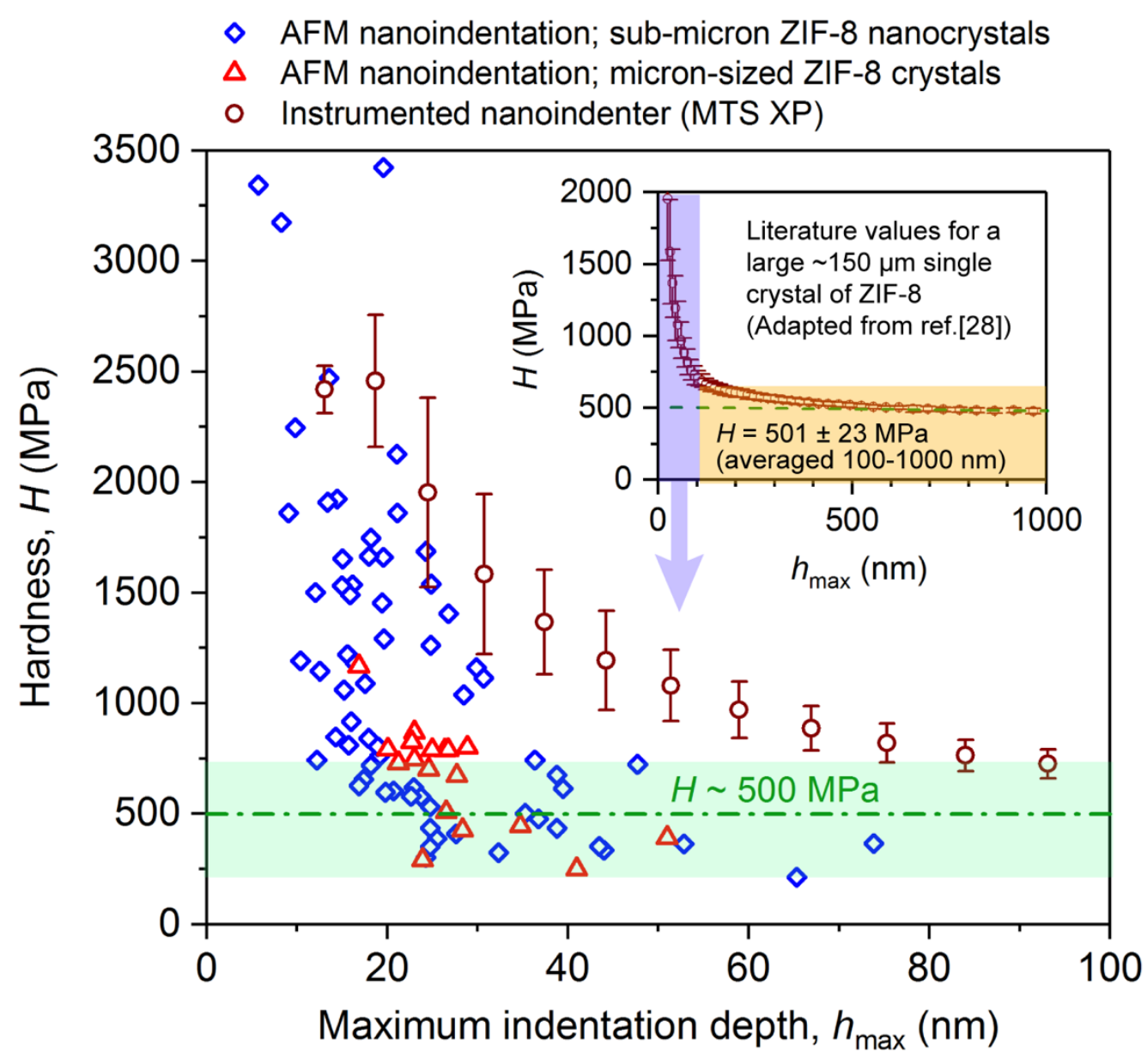

Figure 6. Variation of the hardness of ZIF 8 single crystals as a function of the maximum indentation depth. Blue diamonds represent AFM measurements on nanocrystals of ZIF-8 (size $<500 \mathrm{~nm}$ ), while red triangles are results for micron-sized crystals $(\sim 1-2 \mu \mathrm{m})$, for a total of 97 and 18 indentation experiments respectively. The green dotted line designates the averaged hardness value reported by ref. $^{28}$, but note that this value was obtained using a much larger $(\sim 150 \mu \mathrm{m})$ single crystal of ZIF-8 to enable testing using a standard instrumented nanoindenter. 

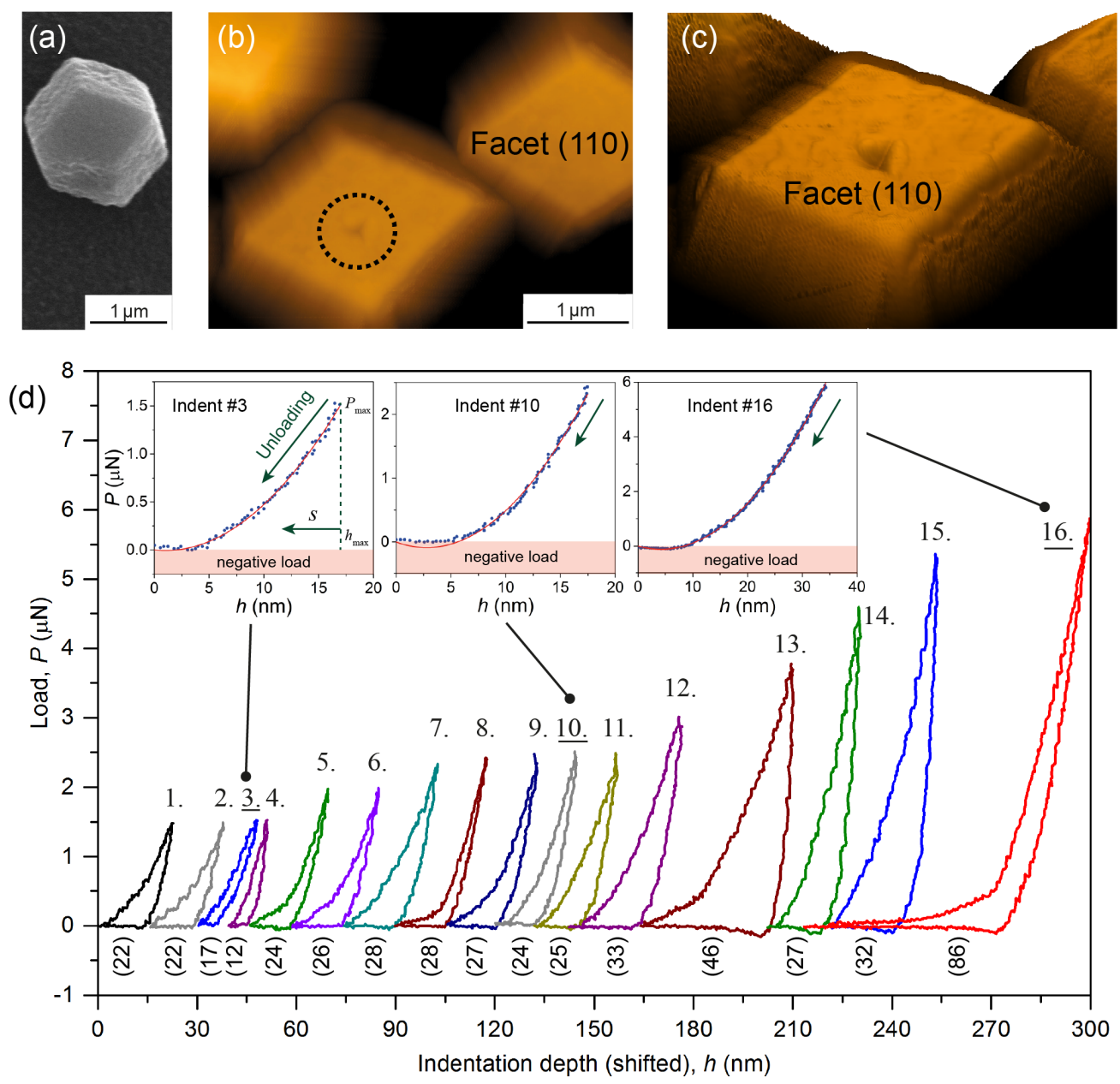

(e)

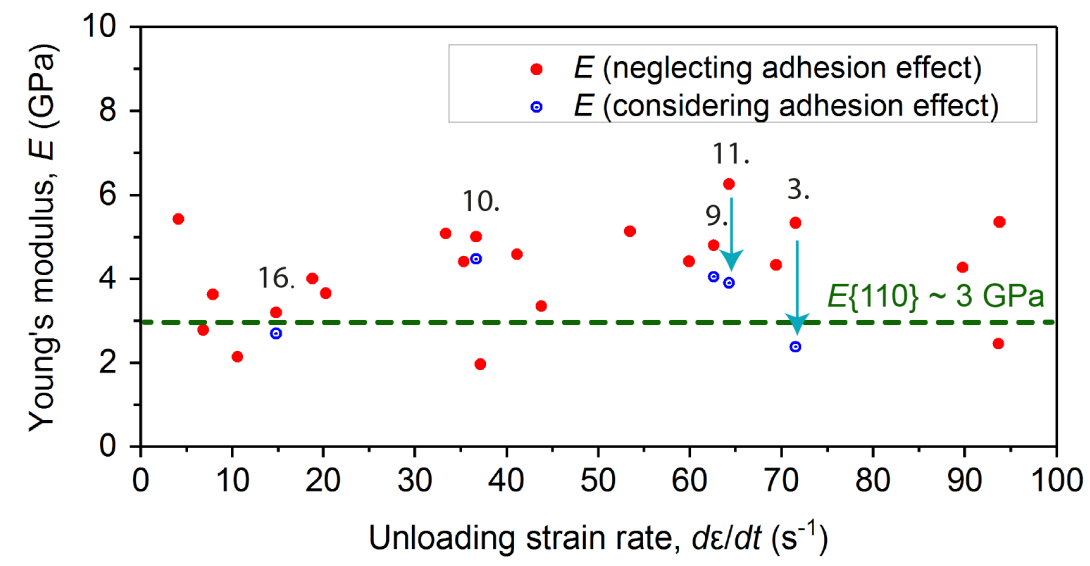

Figure 7. (a) SEM image of an isolated micron-sized single crystal of ZIF-8 used in the AFM nanoindentation study. (b, c) AFM topographic images showing the residual indent on the (110)-oriented crystal facets. This sample corresponds to the $P$ - $h$ curve of indent \#4 shown in 
panel (d). (d) Representative $P$ - $h$ curves of indentation measurements performed on 16 individual crystals, where the numbers in brackets correspond to the maximum indentation depths (in $\mathrm{nm}$ ). The insets show three examples of unloading data with the adhesion segments (negative load), which are fitted using the adhesion model in eq 1. (e) Young's modulus as a function of the unloading strain rate, in which each data point was determined from an individual single crystal whose sample \# is matching the $P$ - $h$ curve in panel (d). The green dotted line indicates the literature value of $E_{\{110\}}$, based on ref. ${ }^{44}$.

\subsection{AFM nanoindentation of micron-sized ZIF-8 crystals}

We conducted AFM nanoindentation on isolated single crystals of ZIF-8, specifically on the $\{110\}$-oriented facets, whose lateral dimensions are $c a .1-2 \mu \mathrm{m}$ as depicted in Figure 7a-c. To put this work into context, while the sub-micron crystals concerned are about $10 \times$ bigger than the nanocrystals we measured, notably they are at least $100 \times$ smaller than that of a ZIF-8 single crystal previously quantifiable via instrumented nanoindentation technique. ${ }^{28}$ Figure $7 \mathrm{~d}$ presents the representative $P$ - $h$ data measured by AFM approach, demonstrating the indentation of 16 individual micron-sized ZIF-8 crystals. It can be seen that the surface penetration depths were approximately $20-30 \mathrm{~nm}$ (satisfying the $10 \%$ depth rule, mitigating substrate effects ${ }^{52}$ ), corresponding to indentation loads measured in the few $\mu \mathrm{N}$ levels. It is worth noting that there is no sign of sample movement by comparing the positions of crystals before and after the indentation process, because crystals are well attached on the substrate after evaporation of solvent and thus immobilized for indentation under AFM. Otherwise, the corresponding force curves will exhibit abnormal behavior, such as "pop-in" phenomenon if crystals are not securely mounted onto substrate. This finding also reflects the advantage of AFM nanoindentation whose loading 
increment is on the $\mathrm{pN}$ level, thereby allowing direct probing of small crystal samples prepared via drop casting.

\subsubsection{Young's modulus and hardness quantification}

The basic methodology we applied to derive the magnitudes of the Young's modulus $(E)$ and hardness $(H)$ properties of micron-sized crystals based on the measured $P$ - $h$ data are identical to the one implemented for analyzing the nanocrystal counterparts. The results of $E$ and $H$ properties obtained as a function of unloading strain rate and indentation depth, are plotted in Figure 7e and Figure 6 respectively. The Young's modulus determined, is in fact: $E_{\{110\}}$, which is in good agreement with the literature value of $\sim 3 \mathrm{GPa}$ (via instrumented nanoindentation of a larger $150 \mu \mathrm{m}$ crystal, Brillouin scattering and DFT calculations ${ }^{44}$ ). Unlike AFM nanoindentation of the nanocrystals (see Figure 5a) that exhibits a strong dependence towards the unloading strain rate (ideally $\dot{\varepsilon}>60 \mathrm{~s}^{-1}$ ), in the case of micron-sized crystals we observed no direct correlation between $E$ and the $\dot{\varepsilon}$ parameter (Figure $7 \mathrm{e}$ ) because of the absence of interparticle sliding prevalent in a polycrystalline sample. Indeed, our results suggest that a $\sim 10 \mathrm{~nm}$ deep indent is sufficient to yield a reliable $E$ value. This finding shows that AFM nanoindentation can be developed into an excellent mechanical characterization tool for probing few-micrometer sized MOF crystals and other nanostructured compounds, which cannot be directly measured by instrumented nanoindentation approach. Furthermore, as shown in Figure 6 the hardness values determined are consistent with the literature ( $H \sim 500 \mathrm{MPa}$, obtained from a large ZIF-8 crystal $){ }^{28}$ albeit with a stronger dependence on the indentation depth. The clear advantage here, however, is the ability to quantify hardness by employing a MOF crystal whose dimension is a hundred times smaller than the minimum size ${ }^{33}$ previously required to enable reliable nanoindentation measurements.

\subsubsection{Contact adhesion forces at the nanoscale}


In this section, we shall consider the additional effects due to contact adhesion forces at the nanometer length scale, established between the indenter tip and crystal surface during the unloading stage of tip withdrawal (from $h_{\max }$ to $h_{\text {final }}$ ). Herein, we implemented the adhesion contact model proposed by Sirghi and Rossi, ${ }^{43}$ who demonstrated that the total interaction force at unloading which accounts for both elastic and adhesive interactions, can be expressed as:

$$
P=\frac{2 E \cdot \tan \theta}{\left(1-v^{2}\right) \pi} \cdot\left(h-h_{\mathrm{final}}\right)^{2}-\gamma_{\mathrm{adhesion}} \cdot \frac{8 \tan \theta}{\pi \cos \theta} \cdot\left(h-h_{\mathrm{final}}\right)
$$

where $\gamma_{\text {adhesion }}$ is termed the thermodynamic work of adhesion ${ }^{59}$ developed between the indenter and the sample surface in contact. More details are presented in the Supporting Information $§ 7$.

We applied eq 1 together with the measured $P$ - $h$ data (Figure $7 \mathrm{~d}$ ) to solve for the magnitudes of $\gamma_{\text {adhesion}}$. For example, we have analyzed the values of $\gamma_{\text {adhesion }}$ for these selected indents: \#3, \#9,\#10,\#11 and \#16, all of which exhibiting a negative load in their unloading test segments (see Figure 7d insets) signifying the effects of adhesion interactions. The insets of Figure 8a-b show the values determined for the thermodynamic work of adhesion; first by letting $\gamma_{\text {adhesion }}$ to be a constant (i.e. independent of depth), ${ }^{43}$ and, subsequently we propose the use of $\gamma_{\text {adhesion }}$ that scales linearly with the surface penetration depth such that $\gamma_{\text {adhesion }} \propto h$. We found that the latter approach results in a more realistic response demonstrating the local variation of adhesion interactions $\left(\sim \mathrm{J} / \mathrm{m}^{2}\right)$ with respect to the indenter position $s$ during tip unloading, see Figure 8b inset. The magnitudes of the thermodynamic work of adhesion determined are broadly found in the range of $\sim 0.4-3.3 \mathrm{~J} / \mathrm{m}^{2}$, whereby its precise value is dependent on the local nature of the indenter-tosurface contact. And interestingly by comparison to the situation where there is no indenter interaction, the "free" surface energy $\left(\gamma_{\mathrm{s}}\right)$ of ZIF-8 has been estimated by DFT to be $\sim 0.28 \mathrm{~J} / \mathrm{m}^{2}{ }^{26}$ Not surprisingly, the thermodynamic work of adhesion we obtained are markedly higher than those 
found on soft polymers (with low Young's modulus and hardness), for example polydimethylsiloxane, PDMS ( $\gamma_{\text {adhesion }}$ is $0.095 \mathrm{~J} / \mathrm{m}^{2}$ and $\left.E \sim 2 \mathrm{MPa}\right)^{43}$ and for a polyimide interface whose $\gamma_{\text {adhesion }} \sim 0.11 \mathrm{~J} / \mathrm{m}^{2}$. $^{60}$

Upon indenter unloading, it follows that the release of the energy of adhesion $W_{\mathrm{a}}$ (negative sign) can be determined from:

$$
W_{\mathrm{a}}=-\gamma_{\mathrm{adhesion}} A_{\text {contact }}
$$

where $A_{\text {contact }}$ is the indenter-to-sample surface contact area.

The relation for an equivalent conical indenter with an included angle of $2 \theta$ is given by:

$$
A_{\text {contact }}=\frac{\pi \cdot \tan \theta}{\cos \theta} \cdot h_{\text {contact }}^{2}
$$

Adhesion energies determined based on the assumptions of, either a constant $\gamma_{\text {adhesion }}$ or a linear correlation of $\gamma_{\text {adhesion }}$, are presented in Figure 8a-b, respectively. The difference between the two approaches becomes even clearer when we compare their first derivatives, i.e. the slope $d W_{\mathrm{a}} / d s$, as demonstrated in the matching Figure 8c-d. Taking the linear correlation of $\gamma_{\text {adhesion }}$, we detected variation in adhesion energy that can be used to elucidate the salient nature of the indenter-to-surface contacts as illustrated in Figure 8d inset, where: (i) the elastic work $\left(W_{\mathrm{el}}\right)>$ plastic work $\left(W_{\mathrm{pl}}\right)$ for $P$ - $h$ curves featuring a predominantly elastic recovery $(e . g$. indents \#3, 16), whereas (ii) $W_{\mathrm{el}}<W_{\mathrm{pl}}$ for an elastic-plastic dominated response experienced in the unloading test segment (e.g. indents \#9, 10,11).

\subsection{Quantitative Measurements of Interfacial Sliding and Fracture Strengths}

We show in Figure 7e that the Young's modulus $(E)$ corrected for adhesion interaction has a relatively lower magnitude than the ones derived using the standard O\&P method. We found that 
when the adhesion forces are hindering the unloading process, the magnitude of $E$ determined may be overestimated by as much as $\sim 1-2 \mathrm{GPa}$; by correcting for the influence of adhesion, we show that this discrepancy could be addressed. The Young's moduli determined, i.e. data points in Figure 7e, with and without considering the contribution from adhesion forces are compared in Table.S1 in Supporting Information.
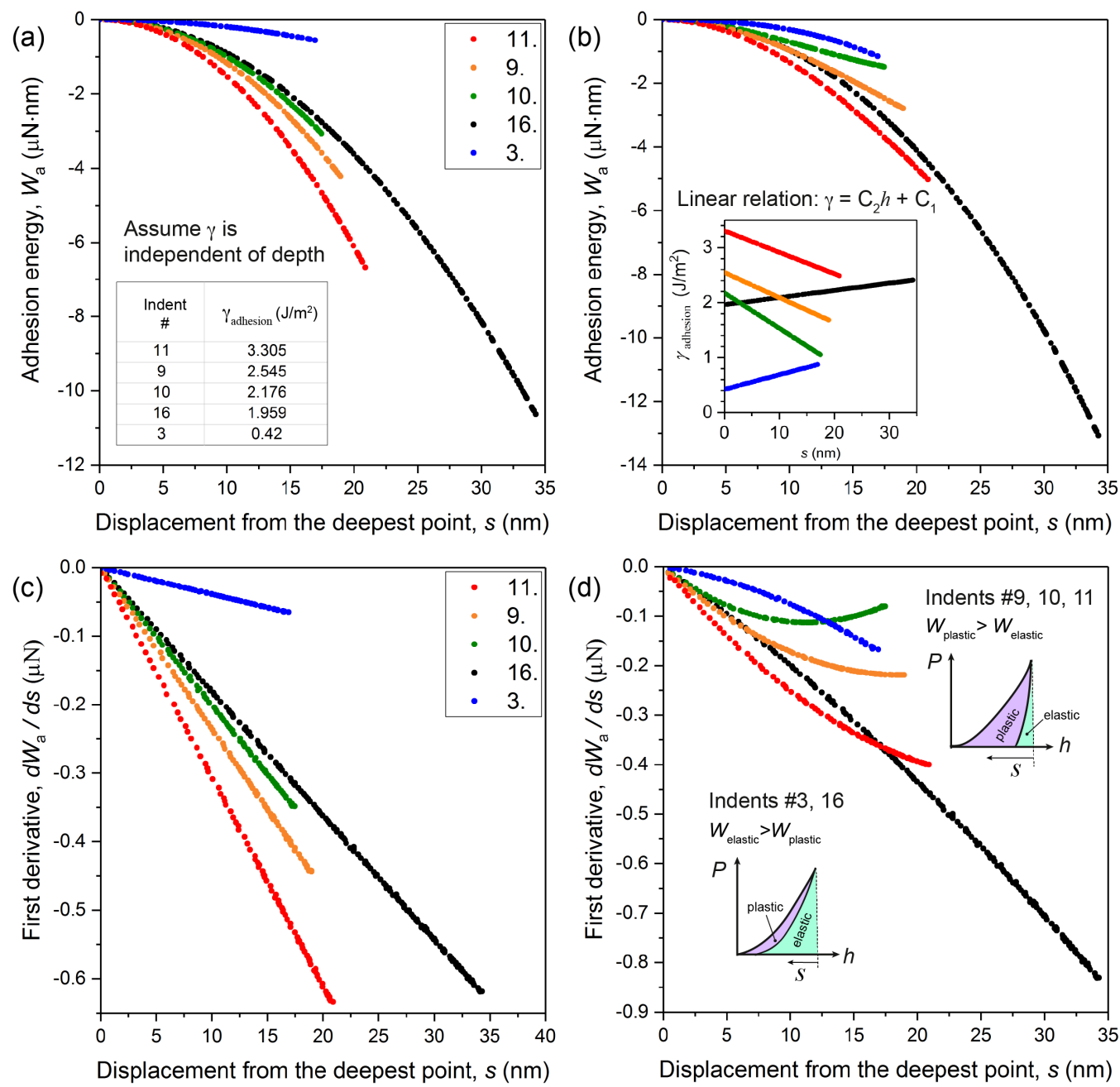

Figure 8. Top panels: $(\mathrm{a}, \mathrm{b})$ Reduction in adhesion energy $\left(-W_{\mathrm{a}}\right)$ as a function of the position of the indenter tip measured from the deepest point of contact, here designated as $s$. The insets show the values of $\gamma_{\text {adhesion }}$, derived from eq 1 , first by letting $\gamma_{\text {adhesion }}$ to be a constant in accordance with 
ref. ${ }^{43}$, and by assuming as a linear function of indentation depth. Bottom panels: (c, d) Plots of $\mathrm{d} W_{\mathrm{a}} / \mathrm{d} s$ as a function of the tip withdrawal distance from the maximum indenter penetration depth. The indentation numbers \# correspond to the $P$ - $h$ curves in Figure 7(d).

We show that the AFM nanoindentation method can be adapted to quantify the interfacial mechanical properties of a polycrystalline thin-film material, comprising a compact "coating" of MOF nanocrystals as shown in Figure 1c. The cube-corner AFM diamond probe can be used to generate significantly deeper indentations, for example with $h_{\max }>100 \mathrm{~nm}$ to be able to measure the collective response of a polycrystalline film. Figure 9 shows the residual impression obtained from a 270-nm deep indentation experiment, it is clear that the response measured here could no longer be associated with that of a single crystal alone. The $P$ - $h$ curve reveals a distinctive "pop-in" effect $^{61}$ that can be attributed to the interfacial sliding or interparticle slippage of adjacent polycrystalline aggregates attributable to shear-induced glide.

We found that such interfacial deformation on a polycrystalline film can yield a much larger surface penetration depth, achieved even with a relatively small indentation load. To illustrate this effect, at the equivalent $P_{\max }$ of $12 \mu \mathrm{N}$, a $60-\mathrm{nm}$ indentation depth was detected without slip (see Figure $3 \mathrm{~b}$ for a single-crystal response) compared to the 270-nm deep indent in Figure 9a associated with a deformation dominated by polycrystalline sliding. Of course, if there is a substantial polycrystalline interfacial contribution to the overall indentation process, it is important to note that the hardness calculated from $H=P_{\max } / A_{\text {contact }}$ will become erroneously low because the contact area becomes significantly larger from interfacial sliding combined with pop-in deformation. 

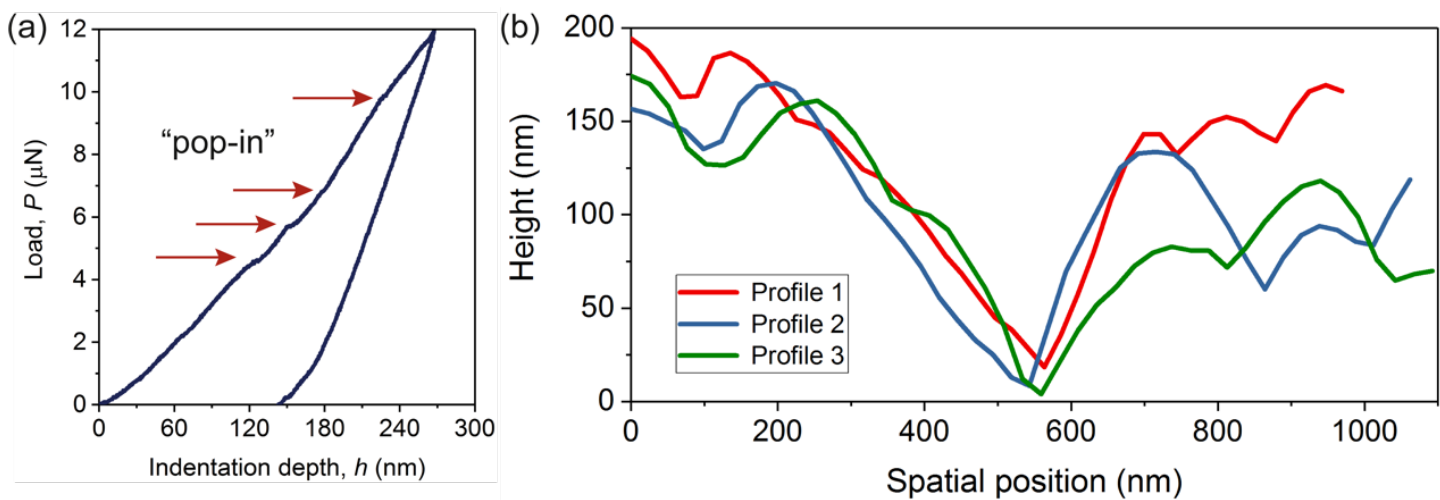

(c)

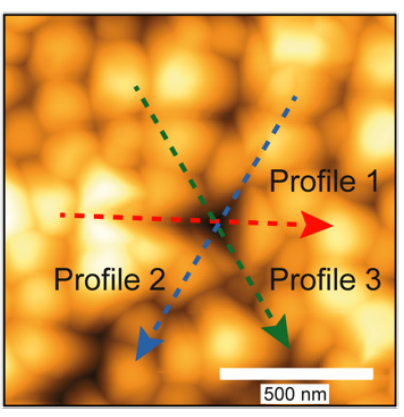

(d)

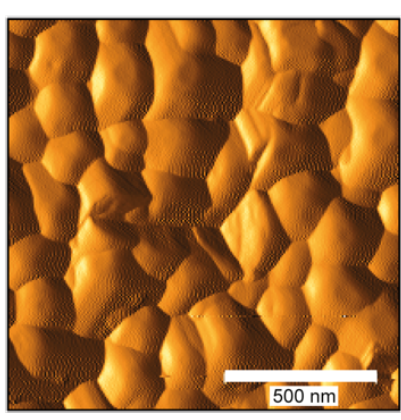

(e)

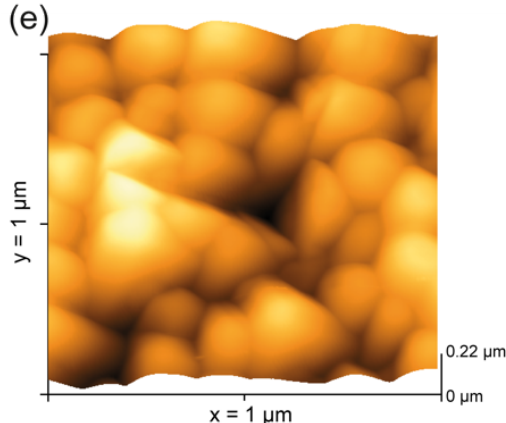

Figure 9. (a) An example of a 270-nm deep indent on the polycrystalline film comprising sub-micron crystals of ZIF-8, where pop-ins can be associated with the interfacial sliding of adjacent nanocrystals occurring in the periphery of the indenter. (b) AFM topographic scans corresponding to the 3-D height images in (c) and (e), height mapping was achieved using the indenter probe. (d) AFM amplitude image revealing the pyramidal-shape of the residual indents.

Here we demonstrate the characterization of the interfacial sliding effects occurring at the grain boundary interface of adjacent polycrystals, when the cube-corner AFM probe was used to indent the polycrystalline film fabricated by drop casting technique. Figure 10a shows three example experiments in which the impressions of the residual indents are visible; we reasoned that the deformation mode associated with interfacial sliding of polycrystals can be represented by the schematic in the bottom panel of Figure $10 \mathrm{~b}$ in which shear-induced sliding and compaction of the nanocrystals will accommodate the large penetration of the tip. Figure 10c-d present the scenario 
whereby the load-displacement curves of indents $P 1$ and $P 2$ indicate a final penetration depth of $\sim 200 \mathrm{~nm}$, but the maximum load of the former indent was relatively lower. Intriguingly, while the maximum load of indent $P 3$ was $\sim 7 \mu \mathrm{N}$ resembling that of indent $P 1$, its maximum indentation depth was nearly three times lower. Our data, therefore, revealed that the exact nature of the sliding deformation underpinning a polycrystalline film (constituting MOF nanocrystals) is a complex phenomenon, which may be triggered by a small external load of an order of just several $\mu \mathrm{N}$. Irregularity detected in the $P$ - $h$ curve can be analyzed by comparing it to the ideal function (without pop-in), with the form of $P(h)=A h^{2}+B h$, given by the red curves in Figure 10c-e. This approach can be used to pinpoint the critical depth $\left(h^{*}\right)$ at which an interfacial sliding deformation occurs, see highlighted regions marked in Figure 10(f-h). We found $h^{*}$ to be ranging from $\sim 50-80 \mathrm{~nm}$ for the polycrystalline film of ZIF-8, which indicates that grain-boundary slippage can be triggered by an indentation depth not exceeding $1 / 3$ of the size of the smallest individual nanocrystals $(\sim 300 \mathrm{~nm})$. 
(a)
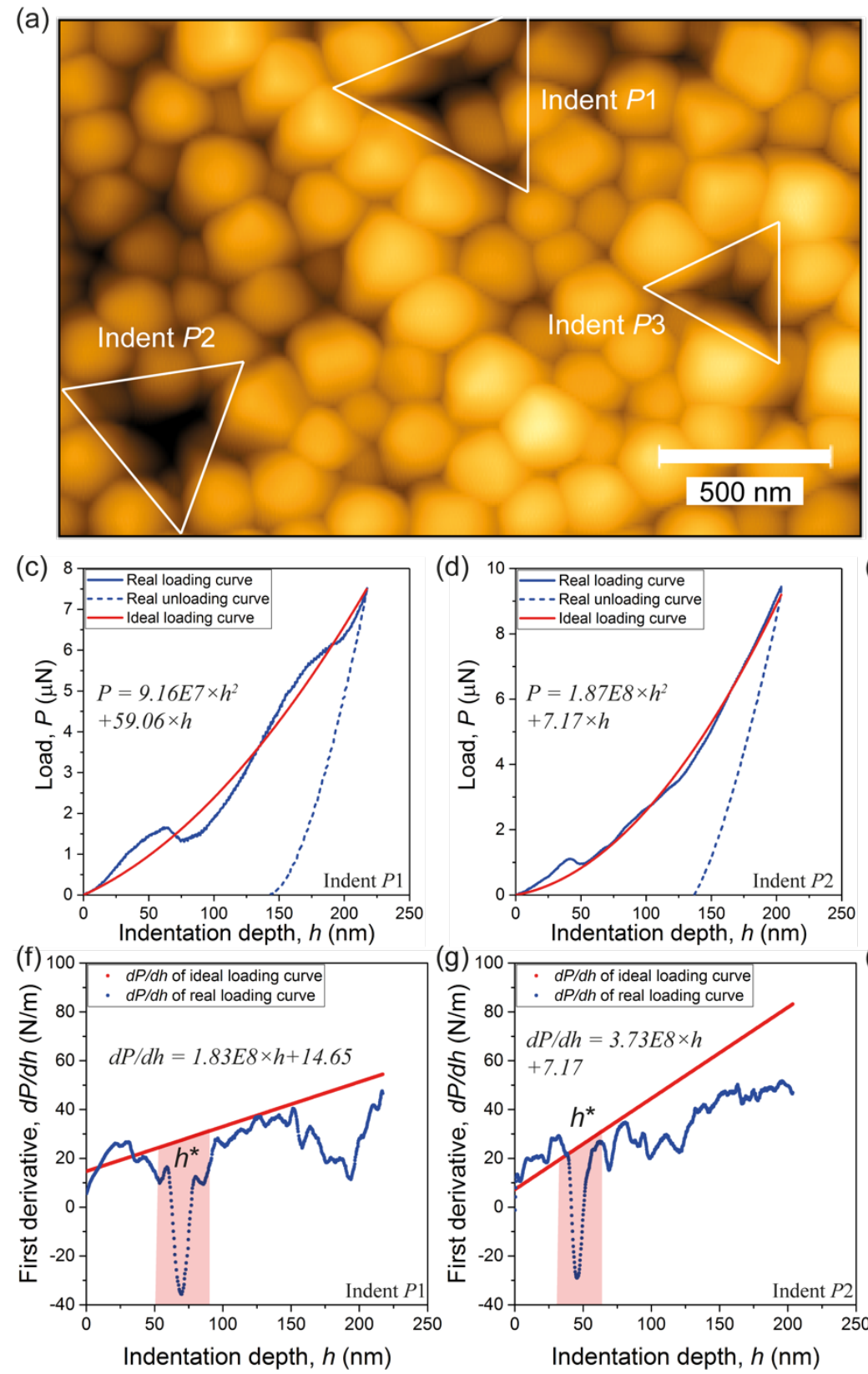

(d) 10 (e)

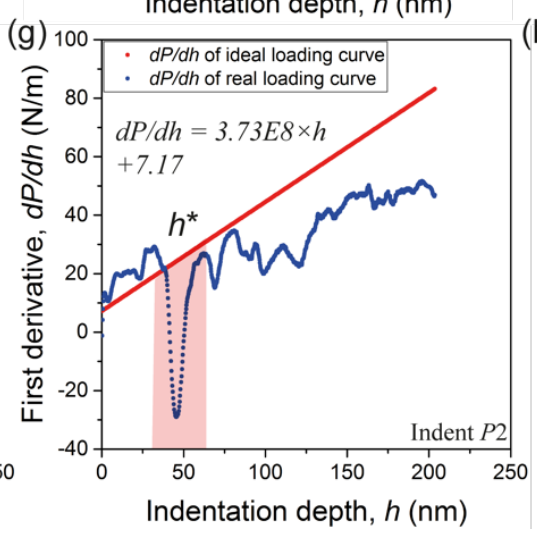

(b)
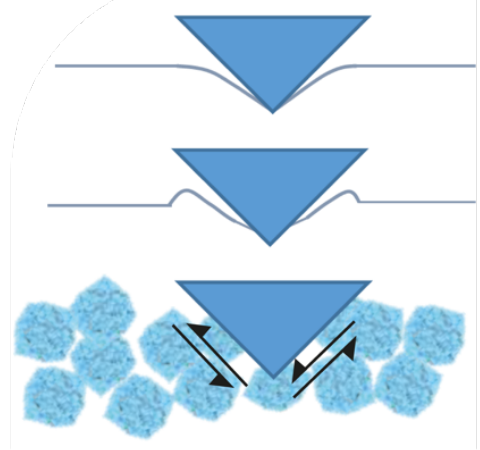
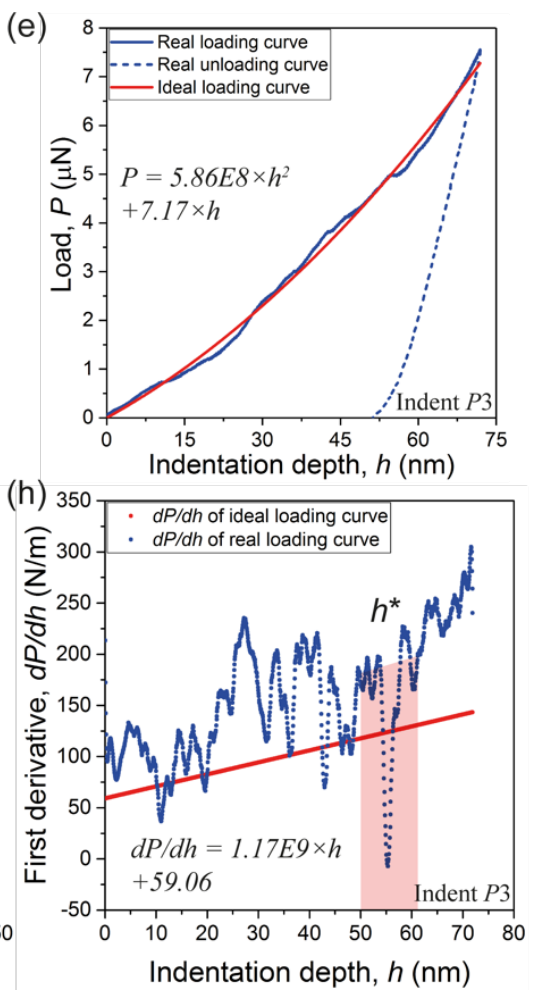

Figure 10. (a) AFM scans showing three residual indents on the polycrystalline film comprising ZIF-8 nanocrystals. (b) Schematic (top to bottom) illustrating the common sink-in or pile-up deformations against the grain-boundary sliding of polycrystals occurring under the indenter tip. (c-e) Force-displacement curves comparing the measured data to the idealized $P$ - $h$ curve in accordance with the relation $P(h)=\mathrm{A} h^{2}+\mathrm{B} h$, where $A$ and $B$ are curve fitting coefficients. The 
indent numbering $P \#$ corresponds to the ones denoted in panel (a). (f-h) Pop-in/sink-in phenomenon where the first major slip events are highlighted, identified by tracking the sharp decline in the contact stiffness, $d P / d h$.

The application of AFM nanoindentation can be extended to characterize the mechanical failure modes of polycrystalline ZIF-8 nanocrystals, the results of which are summarized in Figure 11. Pile-up and sink-in phenomena can be observed in Figs. 11a-b, which we have confirmed by tracking the evolution of the surface topography before and after each indentation measurement. It can be seen that the deformation behaviour is complex, and this will be dependent upon the interfacial strength at the grain boundary between adjacent crystals and the local packing pattern in the vicinity of the indenter tip (Figure 11c-d). The representative failure modes are presented in Figure 11e-f. For instance, the first hump of the loading curve of the indent P5 in Figure 11d was due to the combined effects of crystal fracture and crystal slippage, as evidenced by the AFM image and height profiles in Figure 11b. These failure modes could lead to reduction in the Young modulus and hardness values measured in the experiment. ${ }^{62}$ We have determined the corresponding critical stresses for the four distinctive failure modes A-D, and plotted them as a failure map in Figure 11h. It can be seen that Mode A due to grain boundary slippage could occur at a relatively shallow penetration depth of under $\sim 50 \mathrm{~nm}$, while Mode B is linked to polycrystalline fracture where a failure strength of up to $1 \mathrm{GPa}$ has been determined. Mode $\mathrm{C}$ indicates the accumulated compaction of porous materials during indentation, while Mode D suggests the continuous buckling of bonds along the indentation direction (resembling the buckling response of highly-aligned carbon nanotubes under compression ${ }^{63}$ ). 

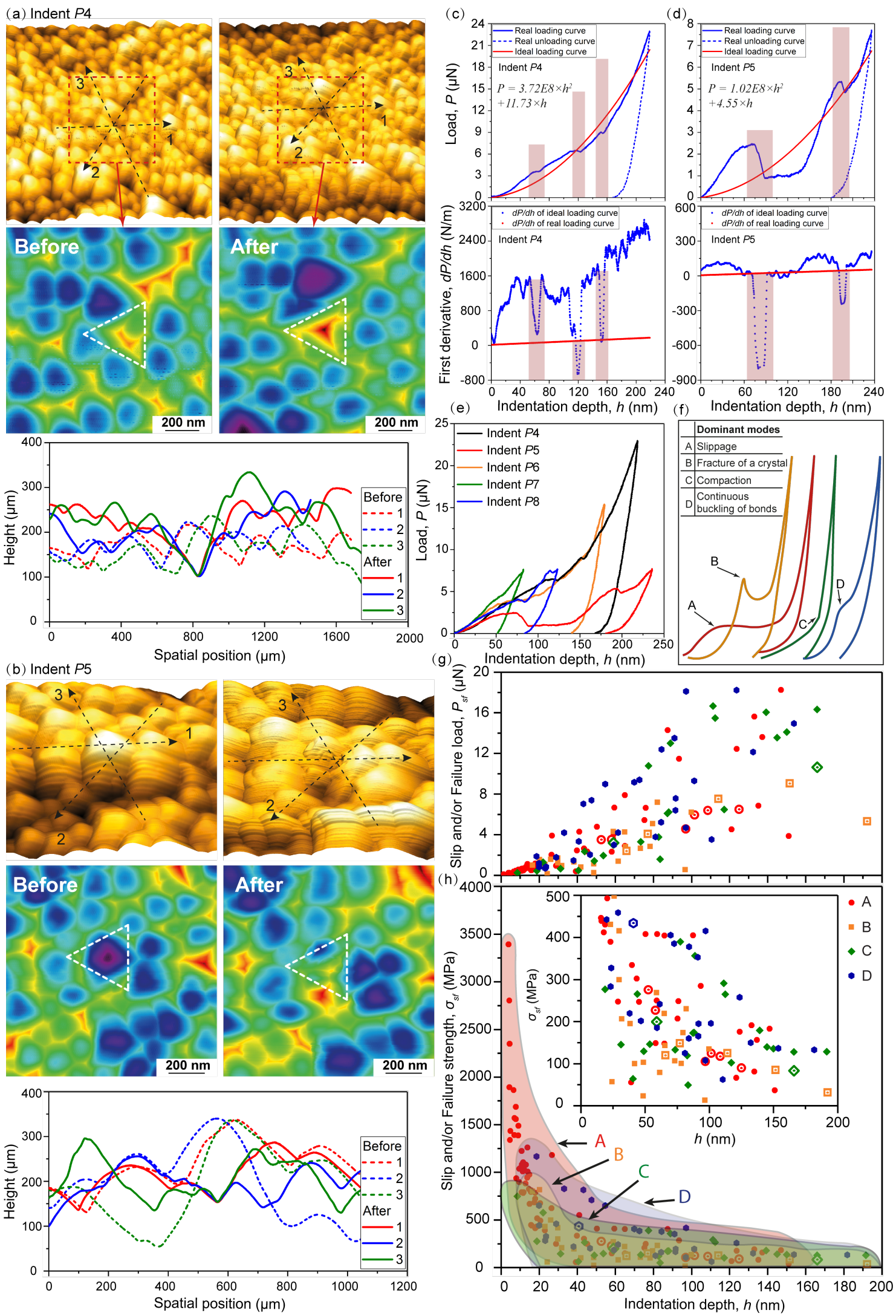
Figure 11. AFM nanoindentation on the polycrystalline ZIF-8 nanocrystals in the form of a thin-film coating: (a) Interfacial slippage-dominant indentation, where the contour map and profiles show the displacement of the surrounding nanocrystals. (b) Crystal facture-dominant indentation, where the contour map and profiles show the fragmentation of a crystal and subsequent slippage. (c, d) Force-displacement curves comparing the measured data to the idealized $P$ - $h$ curve in accordance with the relation $P(h)=\mathrm{A} h^{2}+\mathrm{B} h$, where $A$ and $B$ are curve fitting coefficients. Interfacial slippage and crystal fracture are highlighted, identified by tracking the sharp decline in the contact stiffness, $d P / d h$. (e) Force-displacement curves (indents $P 6-8$ are in Supporting Information: Figure S10-11). (f) Schematic illustrations of the proposed dominant failure modes. (g) Critical loads that resulted in interfacial slip and crystal fracture modes, and, (h) the corresponding stresses of the different failure modes. Note that the open symbols in $(\mathrm{g}, \mathrm{h})$ correspond to the data extracted from indents marked P4-P8 in panel (e).

Likewise, AFM nanoindentation can be used to study failure modes of individual micron-sized ZIF-8 crystals as shown in Figure 12. We observed that the cube-corner diamond indenter (Figure 2) can cause brittle fracture as illustrated for the example of "Crystal A" in Figure 12a-b; this is not surprising because: (i) the pointy tip geometry generates a large stress concentration onto an unconstrained crystal surface to initiate cracking, and (ii) MOFs I $\rightarrow \mathrm{III} \rightarrow \mathrm{IV}$ indicating the corresponding failure loads of each mode. We have performed this type of failure measurements on a total of 40 micron-sized crystals to pinpoint their failure modes, whose results are collated in Figure 12e. These data, in fact, can be better represented as failure strength $\left(\sigma_{\mathrm{f}}\right)$, see Figure 12f, calculated by dividing the individual failure load by its corresponding contact area, $\sigma_{\mathrm{f}}=P_{\mathrm{f}} / A_{\text {contact }}$. 
Figure $12 \mathrm{f}$ shows that the majority of the failure events have been detected at under $\sigma_{\mathrm{y}} \sim 600 \mathrm{MPa}$, which we designated as the "yield strength" of ZIF 8, collectively for Modes I, III, and IV. In fact, the failure strength of Mode-I is lower at under $400 \mathrm{MPa}$. In contrast, Mode-II extends to significantly higher strengths of exceeding $2 \mathrm{GPa}$. We reasoned that the failure mechanisms of the different modes could be explained by considering the local alignment of the indenter axis, in relation to the underlying framework structure of the ZIF-8 crystal. The four plausible failure mechanisms are depicted in Figure $12 \mathrm{~g}$, in which the local indenter axis is pointing normal to the $\{110\}$-oriented facets. We suggest that Mode-I and IV are linked to the rupturing of mIM organic linkages via shearing of the $\mathrm{ZnN}_{4}$ coordination clusters, ${ }^{44}$ a failure mechanism that can be activated at a relatively low level of stress. Mode-II could be explained by buckling of the mIM organic linkages thereby resulting in detection of a distinctive peak load. Mode-III may be associated with breakage of the $\mathrm{ZnN}_{4}-\mathrm{mIM}-\mathrm{ZnN}_{4}$ coordination linkages, followed by sequential pore collapse and material densification (akin to a collapsing foam) ${ }^{64}$ thus causing a sharp rise in indentation force. Nonetheless, confirmation of the precise failure mechanism warrants the application of theoretical approaches, such as large-scale molecular dynamics simulations (see recent examples, refs. ${ }^{24,65}$ ) that are beyond the scope of the current work. Conversely, the failure strengths measured in our study will be useful for the validation of theoretical models as a follow on to this research. 


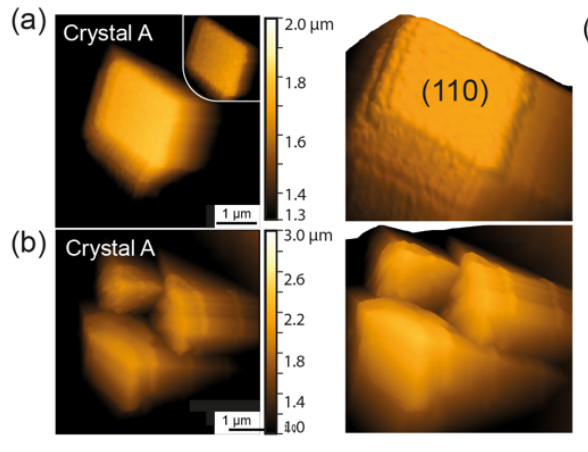

(e)

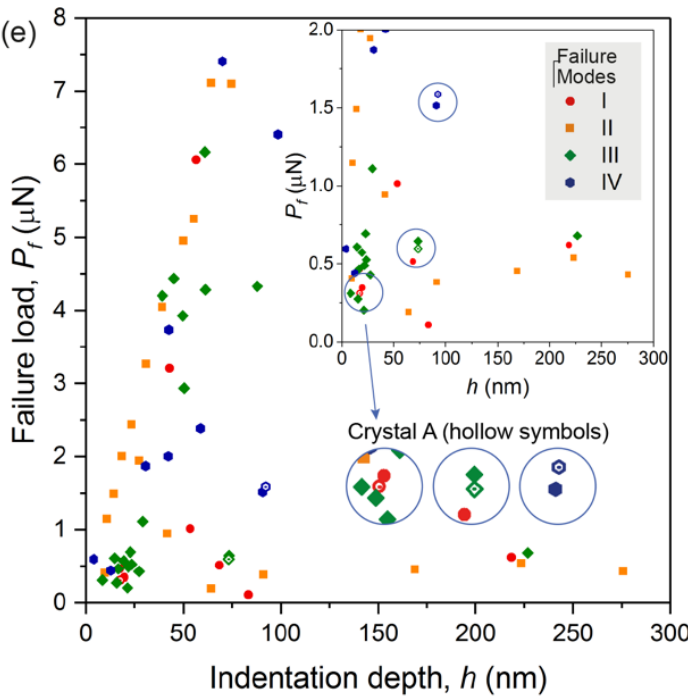

(c)

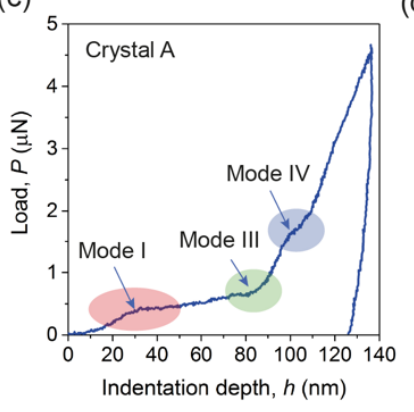

(d)
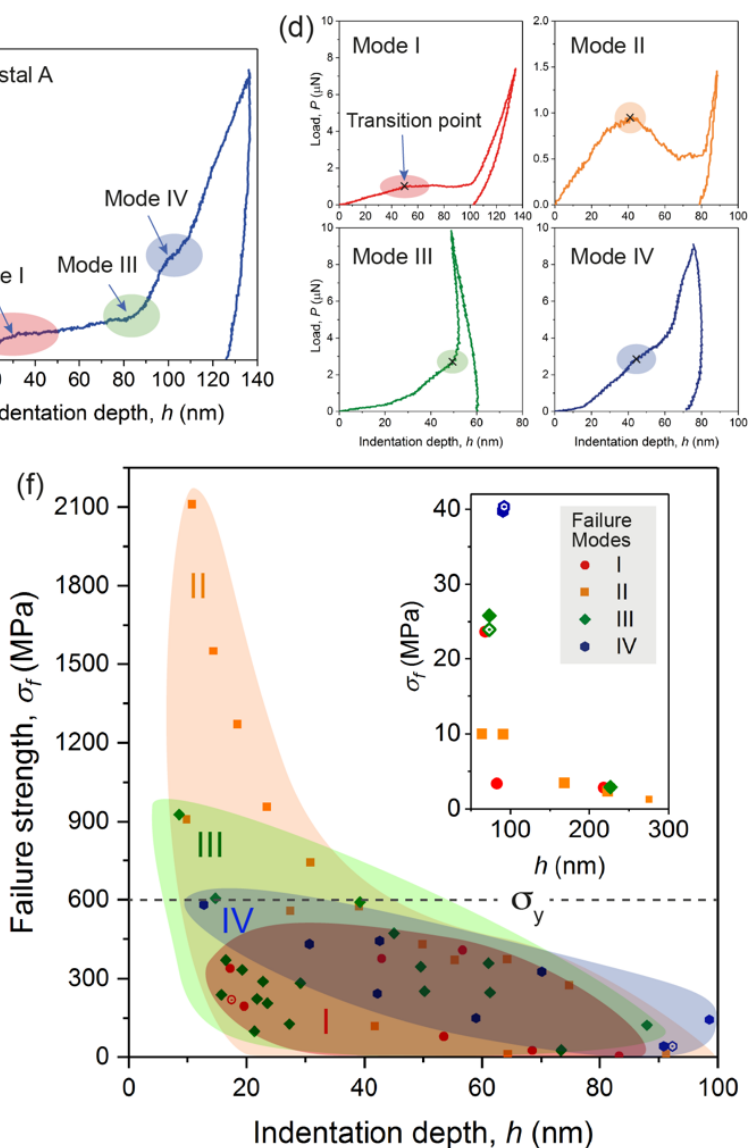
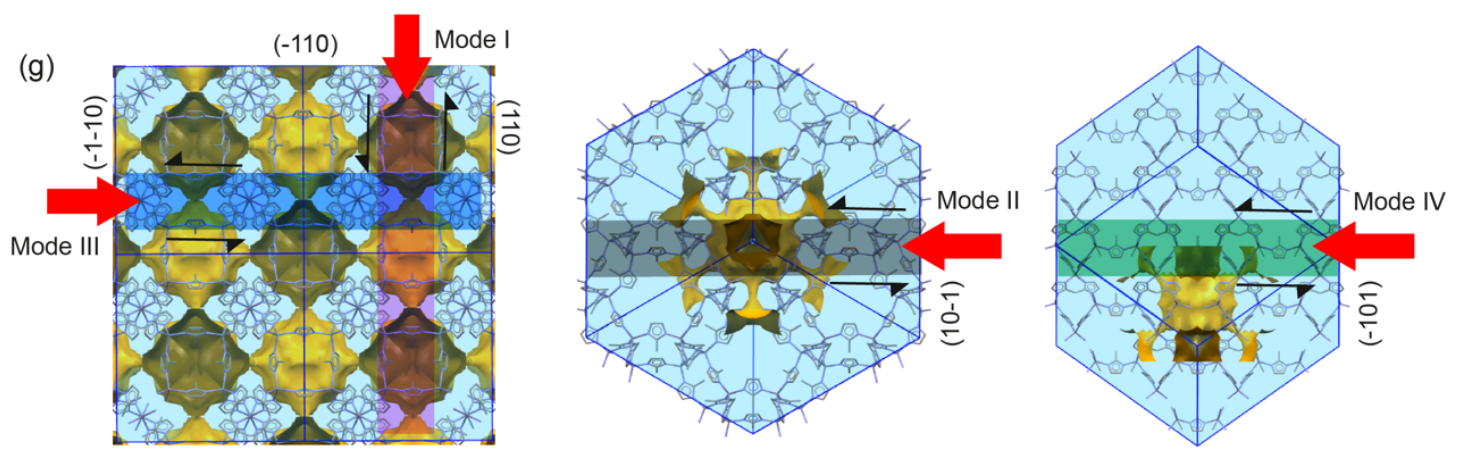

Figure 12. Failure modes of micron-sized crystals of ZIF-8 induced by AFM nanoindentation. (a) AFM topography scans of (a) the micron-sized crystal "A" with the top (110) facet before indentation test, and (b) post-indentation leading to crystal fracture. (c) $P$ - $h$ curve of crystal A, revealing the three different failure modes. (d) Representative $P$ - $h$ curves showing the four failure modes identified in ZIF-8. (e, f) Fracture loads and fracture strengths obtained by indenting 40 
individual samples of micron-sized ZIF-8 crystals, in which the four failure modes are color coded. (g) Proposed failure modes normal to the indented $\{110\}$-oriented crystal facets. The red arrow designates the position of the indenter axis relative to the local crystal structure of ZIF-8. The yellow surfaces represent the porosity (solvent accessible volume) of one cubic unit cell.

\section{Conclusions}

We have demonstrated, for the first time, the efficacy of the AFM nanoindentation approach to achieve quantitative measurements on the fine-scale crystals of a prototypical MOF material: ZIF-8. The primary results are summarized below.

- We have established general AFM nanoindentation methodologies applicable to individual MOF nanocrystals ( $\sim 300$ to $500 \mathrm{~nm}$ ) and isolated micron-sized MOF crystals ( $\sim 1$ to $2 \mu \mathrm{m})$, which to date cannot be characterized accurately using the standard instrumented nanoindenter techniques, because of the very small crystal size.

- AFM nanoindentation enables us to conduct force (few $\mu \mathrm{N}$ ) versus displacement (10s nm) measurements at a significantly higher resolution, from which to derive quantitative mechanical properties such as the Young's modulus and hardness properties. Moreover, AFM allows direct imaging of the residual indent to study surface deformation right after the application of load.

- Importantly, we elucidate how the combination of the AFM instrument parameters, specifically the probe scan rate and the cantilever deflection can be tuned to maximize the unloading strain rate $(\dot{\varepsilon})$. We found that $\dot{\varepsilon}>60 \mathrm{~s}^{-1}$ is necessary to surpass the effects of creep deformation (e.g. in ZIF-8) upon unloading of a compliant sample, to enable accurate determination of the contact stiffness (and subsequently the derived Young's modulus). 
- The high sensitivity of the AFM force transducers $(\mathrm{pN})$ enables the characterization of adhesive force interactions between the indenter tip and the MOF crystal, with which we determined the thermodynamic work of adhesion is of the order of a few $\mathrm{J} / \mathrm{m}^{2}$.

- We demonstrate that the AFM indenter probe can be used to induce grain-boundary interfacial sliding of a polycrystalline MOF film to investigate the pop-in and slippage phenomena.

- We show how AFM nanoindentation can be employed to probe the local failure modes of micron-sized MOF crystals, and to enable quantitative measurements of fracture strengths of tiny crystals.

- The methodologies described in this study are applicable to the fine-scale mechanical characterization of other porous MOFs, 3-D framework crystals, 2-D nanosheet materials and polycrystalline films. For example, we are currently extending this AFM nanoindentation technique to quantify the mechanical properties of 2-D nanosheets of CuBDC ${ }^{66}$ HKUST-1 crystals and monoliths, covalent organic frameworks $(\mathrm{COFs})^{67}$ and a number of MOF-polymer nanocomposites; ${ }^{.6-69}$ the results of which will be the subject of forthcoming publications. 


\section{ASSOCIATED CONTENTS}

\section{Supporting Information:}

1. Characterization of the size of sub-micron ZIF-8 crystals from AFM images

2. Characterization of the geometry of the cube-corner AFM indenter tip

3. Loading scheme of the Veeco Dimension 3100 AFM instrument

4. AFM nanoindentation of sub-micron sized crystals of ZIF-8 (nanocrystals)

5. Creep deformation at the point of indenter unloading

6. AFM nanoindentation on micron-sized ZIF-8 crystals

7. Contact adhesion forces at the nanoscale

8. Fracture studies using AFM nanoindentation

9. Oliver and Pharr (O\&P) Method

10. Key factors that could introduce errors in the AFM nanoindentation measurements

\section{AUTHOR INFORMATION}

\section{Corresponding Author}

*Jin-Chong Tan, Multifunctional Materials \& Composites (MMC) Laboratory, Department of Engineering Science, University of Oxford, Parks Road, Oxford OX1 3PJ, United Kingdom

Email: jin-chong.tan@eng.ox.ac.uk

\section{Author Contributions}

JCT conceived the project, ZXZ performed the experiments and analyzed the data with input from JCT. The manuscript was written through contributions of all authors. All authors have given approval to the final version of the manuscript.

\section{ACKNOWLEDGMENTS}

J.C.T. would like to acknowledge the Engineering and Physical Sciences Research Council, EPSRC RCUK (EP/N014960/1) for research funding.Z.X.Z. is grateful to Dr. Gavin Stenning and 
Dr. Marek Jura at the R53 Materials Characterization Lab (ISIS Rutherford Appleton Laboratory, Oxfordshire), for the provision of X-ray diffraction facilities. We thank the Research Complex at Harwell (RCaH) for access to the advanced materials characterization suite.

\section{REFERENCES}

1. Tan, J. C.; Cheetham, A. K. Mechanical Properties of Hybrid Inorganic-Organic Framework Materials: Establishing Fundamental Structure-Property Relationships. Chem. Soc. Rev. 2011, 40, 1059-1080.

2. Allendorf, M. D.; Stavila, V. Crystal engineering, structure-function relationships, and the future of metal-organic frameworks. CrystEngComm 2015, 17, 229-246.

3. Howarth, A. J.; Liu, Y. Y.; Li, P.; Li, Z. Y.; Wang, T. C.; Hupp, J.; Farha, O. K. Chemical, thermal and mechanical stabilities of metal-organic frameworks. Nat. Rev. Mater. 2016, l, 15018 .

4. Tan, J. C.; Civalleri, B. Metal-Organic Frameworks and Hybrid Materials: From Fundamentals to Applications. CrystEngComm 2015, 17, 197-198.

5. Zhu, Q. L.; Xu, Q. Metal-organic framework composites. Chem. Soc. Rev. 2014, 43, 54685512 .

6. Phan, A.; Doonan, C. J.; Uribe-Romo, F. J.; Knobler, C. B.; O'Keeffe, M.; Yaghi, O. M. Synthesis, structure and carbon dioxide capture properties of zeolitic imidazolate frameworks. Acc. Chem. Res. 2010, 43, 58-67.

7. Valenzano, L.; Civalleri, B.; Chavan, S.; Bordiga, S.; Nilsen, M. H.; Jakobsen, S.; Lillerud, K. P.; Lamberti, C. Disclosing the Complex Structure of UiO-66 Metal Organic Framework: A Synergic Combination of Experiment and Theory. Chem. Mater. 2011, 23, 1700-1718.

8. Ryder, M. R.; Civalleri, B.; Tan, J. C. Isoreticular zirconium-based metal-organic frameworks: discovering mechanical trends and elastic anomalies controlling chemical structure stability. Phys. Chem. Chem. Phys. 2016, 18, 9079-9087.

9. Ferey, G.; Serre, C. Large breathing effects in three-dimensional porous hybrid matter: facts, analyses, rules and consequences. Chem. Soc. Rev. 2009, 38, 1380-1399.

10. Ryder, M. R.; Civalleri, B.; Cinque, G.; Tan, J. C. Discovering connections between terahertz vibrations and elasticity underpinning the collective dynamics of the HKUST-1 metalorganic framework. CrystEngComm 2016, 18, 4303-4312. 
11. Díaz-García, M.; Mayoral, Á.; Díaz, I.; Sánchez-Sánchez, M. Nanoscaled M-MOF-74 Materials Prepared at Room Temperature. Cryst. Growth Des. 2014, 14, 2479-2487.

12. Wharmby, M. T.; Mowat, J. P.; Thompson, S. P.; Wright, P. A. Extending the pore size of crystalline metal phosphonates toward the mesoporous regime by isoreticular synthesis. J. Am. Chem. Soc. 2011, 133, 1266-1269.

13. Caro, J. Are MOF membranes better in gas separation than those made of zeolites? Curr. Opin. Chem. Eng. 2011, 1, 77-83.

14. Bao, Z. B.; Chang, G. G.; Xing, H. B.; Krishna, R.; Ren, Q. L.; Chen, B. L. Potential of microporous metal-organic frameworks for separation of hydrocarbon mixtures. Energy Environ. Sci. 2016, 9, 3612-3641.

15. Ryder, M. R.; Tan, J. C. Nanoporous Metal-Organic Framework Materials for Smart Applications. Mater. Sci. Tech. 2014, 30, 1598-1612.

16. Usman, M.; Mendiratta, S.; Lu, K. L. Metal-Organic Frameworks: New Interlayer Dielectric Materials. ChemElectroChem 2015, 2, 786-788.

17. Dolgopolova, E. A.; Shustova, N. B. Metal-organic framework photophysics: Optoelectronic devices, photoswitches, sensors, and photocatalysts. MRS Bull. 2016, 41, 890896.

18. Li, W.; Henke, S.; Cheetham, A. K. Research Update: Mechanical properties of metalorganic frameworks - Influence of structure and chemical bonding. APL Mater. 2014, 2, 123902.

19. Ryder, M. R.; Tan, J. C. Explaining the mechanical mechanisms of zeolitic metal-organic frameworks: revealing auxeticity and anomalous elasticity. Dalton Trans. 2016, 45, 4154-4161.

20. Wu, H.; Yildirim, T.; Zhou, W. Exceptional Mechanical Stability of Highly Porous Zirconium Metal-Organic Framework UiO-66 and Its Important Implications. J. Phys. Chem. Lett. 2013, 4, 925-930.

21. Ortiz, A. U.; Boutin, A.; Fuchs, A. H.; Coudert, F.-X. Anisotropic Elastic Properties of Flexible Metal-Organic Frameworks: How Soft are Soft Porous Crystals? Phys. Rev. Lett. 2012, $109,195502$.

22. Tan, J. C.; Civalleri, B.; Erba, A.; Albanese, E. Quantum mechanical predictions to elucidate the anisotropic elastic properties of zeolitic imidazolate frameworks: ZIF-4 vs. ZIF-zni.

CrystEngComm 2015, 17, 375-382.

23. Marmier, A.; Evans, K. E. Flexibility in MOFs: do scalar and group-theoretical counting rules work? Dalton Trans. 2016, 45, 4360-4369.

24. Banlusan, K.; Antillon, E.; Strachan, A. Mechanisms of Plastic Deformation of MetalOrganic Framework-5. J. Phys. Chem. C 2015, 119, 25845-25852. 
25. Ortiz, A. U.; Boutin, A.; Fuchs, A. H.; Coudert, F.-X. Investigating the Pressure-Induced Amorphization of Zeolitic Imidazolate Framework ZIF-8: Mechanical Instability Due to Shear Mode Softening. J. Phys. Chem. Lett. 2013, 4, 1861-1865.

26. Hegde, V. I.; Tan, J. C.; Waghmare, U. V.; Cheetham, A. K. Stacking Faults and Mechanical Behavior Beyond the Elastic Limit of an Imidazole-Based Metal Organic Framework: ZIF-8. J. Phys. Chem. Lett. 2013, 4, 3377-3381.

27. Bahr, D. F.; Reid, J. A.; Mook, W. M.; Bauer, C. A.; Stumpf, R.; Skulan, A. J.; Moody, N. R.; Simmons, B. A.; Shindel, M. M.; Allendorf, M. D. Mechanical properties of cubic zinc carboxylate IRMOF-1 metal-organic framework crystals. Phys. Rev. B 2007, 76, 184106.

28. Tan, J. C.; Bennett, T. D.; Cheetham, A. K. Chemical structure, network topology, and porosity effects on the mechanical properties of Zeolitic Imidazolate Frameworks. Proc. Natl. Acad. Sci. USA 2010, 107, 9938-9943.

29. Henke, S.; Li, W.; Cheetham, A. K. Guest-dependent mechanical anisotropy in pillaredlayered soft porous crystals - a nanoindentation study. Chem. Sci. 2014, 5, 2392-2397.

30. Reddy, C. M.; Krishna, G. R.; Ghosh, S. Mechanical properties of molecular crystalsapplications to crystal engineering. CrystEngComm 2010, 12, 2296-2314.

31. Kiran, M. S. R. N.; Varughese, S.; Reddy, C. M.; Ramamurty, U.; Desiraju, G. R. Mechanical Anisotropy in Crystalline Saccharin: Nanoindentation Studies. Cryst. Growth Des. 2010, 10, 4650-4655.

32. Gao, H.; Wei, W.; Dong, L.; Feng, G.; Jiang, X.; Wu, R.; Lin, Z.; Li, W. Enhanced Framework Rigidity of a Zeolitic Metal-Azolate via Ligand Substitution. Crystals 2017, 7, 1-11.

33. Ramamurty, U.; Jang, J. I. Nanoindentation for probing the mechanical behavior of molecular crystals-a review of the technique and how to use it. CrystEngComm 2014, 16, 12-23.

34. Stock, N.; Biswas, S. Synthesis of metal-organic frameworks (MOFs): routes to various MOF topologies, morphologies, and composites. Chem. Rev. 2012, 112, 933-969.

35. Eslava, S.; Zhang, L.; Esconjauregui, S.; Yang, J.; Vanstreels, K.; Baklanov, M. R.; Saiz, E. Metal-Organic Framework ZIF-8 Films As Low-к Dielectrics in Microelectronics. Chem. Mater. 2013, 25, 27-33.

36. Van de Voorde, B.; Ameloot, R.; Stassen, I.; Everaert, M.; De Vos, D.; Tan, J. C. Mechanical Properties of Electrochemically Synthesised Metal-Organic Framework Thin Films. J. Mater. Chem. B 2013, 1, 7716-7724.

37. Bundschuh, S.; Kraft, O.; Arslan, H. K.; Gliemann, H.; Weidler, P. G.; Wöll, C. Mechanical properties of metal-organic frameworks: An indentation study on epitaxial thin films. Appl. Phys. Lett. 2012, 101, 101910. 
38. Stassen, I.; Styles, M.; Van Assche, T.; Campagnol, N.; Fransaer, J.; Denayer, J.; Tan, J. C.; Falcaro, P.; De Vos, D.; Ameloot, R. Electrochemical Film Deposition of the Zirconium MetalOrganic Framework UiO-66 and Application in a Miniaturized Sorbent Trap. Chem. Mater. 2015, 27, 1801-1807.

39. Buchan, I.; Ryder, M. R.; Tan, J. C. Micromechanical Behavior of Polycrystalline MetalOrganic Framework Thin Films Synthesized by Electrochemical Reaction. Cryst. Growth Des. 2015, 15, 1991-1999.

40. Ferencz, R.; Sanchez, J.; Blümich, B.; Herrmann, W. AFM nanoindentation to determine Young's modulus for different EPDM elastomers. Polymer Test. 2012, 31, 425-432.

41. Kontomaris, S. V.; Stylianou, A. Atomic force microscopy for university students: applications in biomaterials. Eur. J. Phys. 2017, 38, 033003.

42. Cohen, S. R.; Kalfon-Cohen, E. Dynamic nanoindentation by instrumented nanoindentation and force microscopy: a comparative review. Beilstein J. Nanotechnol. 2013, 4, 815-833.

43. Sirghi, L.; Rossi, F. Adhesion and elasticity in nanoscale indentation. Appl. Phys. Lett. 2006, $89,243118$.

44. Tan, J. C.; Civalleri, B.; Lin, C. C.; Valenzano, L.; Galvelis, R.; Chen, P. F.; Bennett, T. D.; Mellot-Draznieks, C.; Zicovich-Wilson, C. M.; Cheetham, A. K. Exceptionally Low Shear Modulus in a Prototypical Imidazole-Based Metal-Organic Framework. Phys. Rev. Lett. 2012, $108,095502$.

45. Villarrubia, J. S. Algorithms for scanned probe microscope image simulation, surface reconstruction, and tip estimation. J. Res. Natl. Inst. Stand. Technol. 1997, 102, 425-454.

46. Dongmo, L. S.; Villarrubia, J. S.; Jones, S. N.; Renegar, T. B.; Postek, M.; Song, J. F. Experimental test of blind tip reconstruction for scanning probe microscopy. Ultramicroscopy 2000, $85,141-153$.

47. Necas, D.; Klapetek, P. Gwyddion: an open-source software for SPM data analysis. Cent. Eur. J. Phys. 2012, 10, 181-188.

48. Oliver, W. C.; Pharr, G. M. Measurement of hardness and elastic modulus by instrumented indentation: Advances in understanding and refinements to methodology. J. Mater. Res. 2004, 19, 3-20.

49. Sweers, K. K.; Bennink, M. L.; Subramaniam, V. Nanomechanical properties of single amyloid fibrils. J. Phys.: Condens. Matter. 2012, 24, 243101.

50. Tan, J. C.; Merrill, C. A.; Orton, J. B.; Cheetham, A. K. Anisotropic Mechanical Properties of Polymorphic Hybrid Inorganic-Organic Framework Materials with Different

Dimensionalities. Acta. Mater. 2009, 57, 3481-3496. 
51. Briscoe, B. J.; Fiori, L.; Pelillo, E. Nano-indentation of polymeric surfaces. J. Phys. D-Appl. Phys. 1998, 31, 2395-2405.

52. Fisher-Cripps, A. C. Nanoindentation. 2nd ed.; Springer, New York: 2004.

53. Tranchida, D.; Kiflie, Z.; Acierno, S.; Piccarolo, S. Nanoscale mechanical characterization of polymers by atomic force microscopy (AFM) nanoindentations: viscoelastic characterization of a model material. Meas. Sci. Technol. 2009, 20, 095702.

54. Moeller, G. AFM Nanoindentation of Viscoelastic Materials with Large End-Radius Probes. J. Polym. Sci., Part B: Polym. Phys. 2009, 47, 1573-1587.

55. Lucas, M.; Gall, K.; Riedo, E. Tip size effects on atomic force microscopy nanoindentation of a gold single crystal. J. Appl. Phys. 2008, 104, 113515.

56. Pugno, N. M. A general shape/size-effect law for nanoindentation. Acta. Mater. 2007, 55, 1947-1953.

57. Durst, K.; Göken, M.; Pharr, G. M. Indentation size effect in spherical and pyramidal indentations. J. Phys. D-Appl. Phys. 2008, 41, 074005.

58. Tan, J. C.; Furman, J. D.; Cheetham, A. K. Relating Mechanical Properties and Chemical Bonding in An Inorganic-Organic Framework Material: A Single-Crystal Nanoindentation Study. J. Am. Chem. Soc. 2009, 131, 14252-14254.

59. Williamson, D. M.; Hamilton, N. R.; Palmer, S. J. P.; Jardine, A. P.; Leppard, C.

Thermodynamic work of adhesion measurements of polymer bonded explosive constituents via the Wilhelmy plate technique and their application to AFM pull-off experiments. J. Phys. Conf. Ser. 2014, 500, 112068.

60. Okamatsu, T.; Yasuda, Y.; Ochi, M. Thermodynamic work of adhesion and peel adhesion energy of dimethoxysilyl-terminated polypropylene oxide/epoxy resin system jointed with polymeric substrates. J. Appl. Polym. Sci. 2001, 80, 1920-1930.

61. Tan, J. C.; Saines, P. J.; Bithell, E. G.; Cheetham, A. K. Hybrid Nanosheets of an InorganicOrganic Framework Material: Facile Synthesis, Structure, and Elastic Properties. ACS Nano 2012, 6, 615-621.

62. Chen, Y.; Bakshi, S. R.; Agarwal, A. Intersplat Friction Force and Splat Sliding in a PlasmaSprayed Aluminum Alloy Coating during Nanoindentation and Microindentation. ACS Appl. Mater. Interfaces 2009, 1, 235-238.

63. Maschmann, M. R.; Zhang, Q. H.; Wheeler, R.; Du, F.; Dai, L. M.; Baur, J. In situ SEM Observation of Column-like and Foam-like CNT Array Nanoindentation. ACS Appl. Mater. Interfaces 2011, 3, 648-653.

64. Marx, J.; Rabiei, A. Overview of Composite Metal Foams and Their Properties and Performance. Adv. Eng. Mater. 2017, 1600776. 
65. Banlusan, K.; Strachan, A. Shockwave Energy Dissipation in Metal-Organic Framework MOF-5. J. Phys. Chem. C 2016, 120, 12463-12471.

66. Rodenas, T.; Luz, I.; Prieto, G.; Seoane, B.; Miro, H.; Corma, A.; Kapteijn, F.; Llabres i Xamena, F. X.; Gascon, J. Metal-organic framework nanosheets in polymer composite materials for gas separation. Nat. Mater. 2015, 14, 48-55.

67. Ding, S.-Y.; Wang, W. Covalent organic frameworks (COFs): from design to applications. Chem. Soc. Rev. 2013, 42, 548-568.

68. Mahdi, E. M.; Tan, J. C. Mixed-matrix membranes of zeolitic imidazolate framework (ZIF8)/Matrimid nanocomposite: Thermo-mechanical stability and viscoelasticity underpinning membrane separation performance. J. Membr. Sci. 2016, 498, 276-290.

69. Flyagina, I. S.; Mahdi, E. M.; Titov, K.; Tan, J. C. Thermo-mechanical properties of mixedmatrix membranes encompassing zeolitic imidazolate framework-90 and polyvinylidine difluoride: ZIF-90/PVDF nanocomposites. APL Mater. 2017, 5, 086104. 


\section{TOC graphic}

Nanoindentation under the atomic force microscope (AFM) to measure the elastic, plastic, adhesion, and fracture properties of small crystals of MOFs.

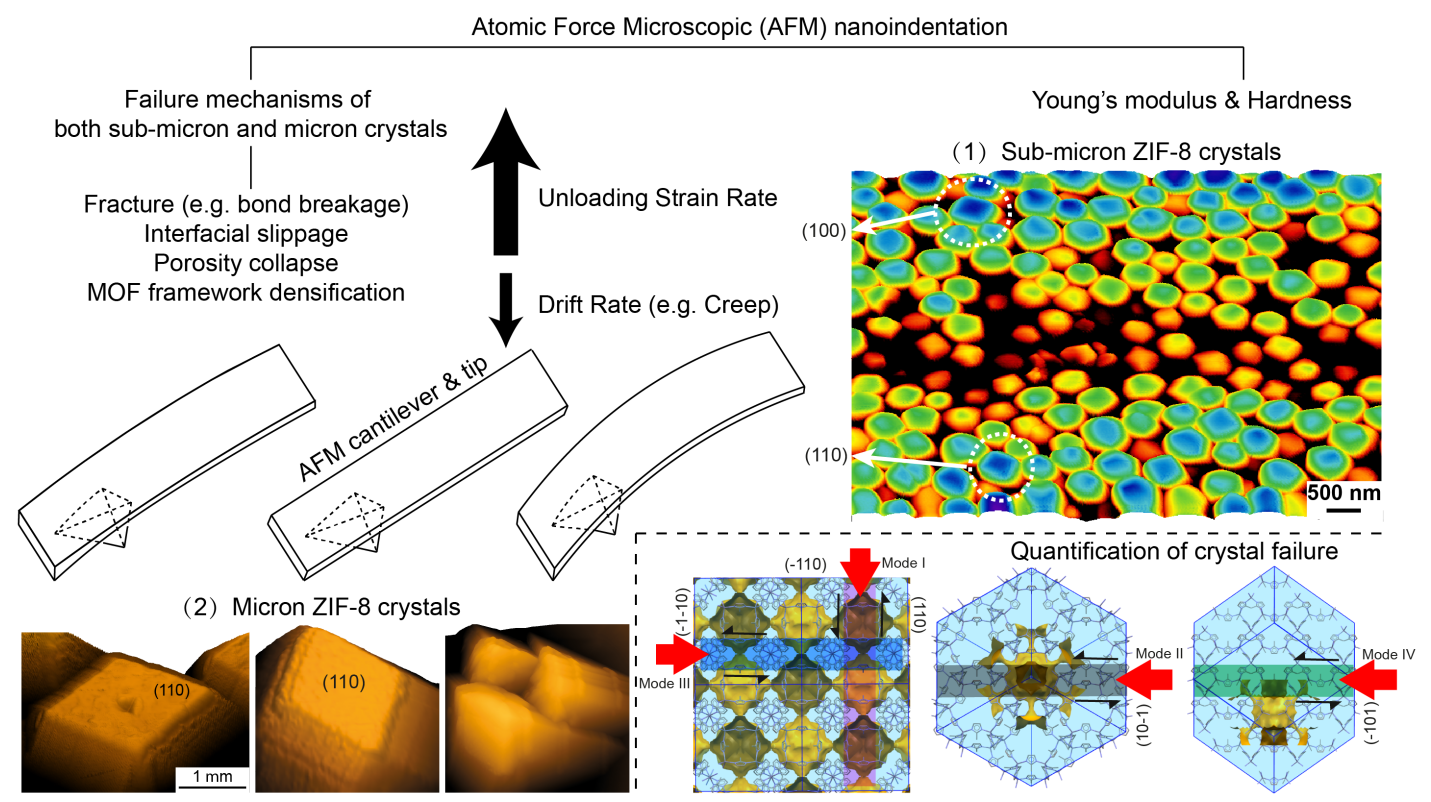

